\title{
The Earth's Free Oscillations and the Differential Rotation of the Inner Core
}

\author{
Gabi Laske and Guy Masters \\ I.G.P.P., U.C.S.D., La Jolla, California 92093-0225, U.S.A.
}

\begin{abstract}
Differential rotation of the inner core has been inferred by several body-wave studies with most agreeing that a superrotation may exist with a rate between $0.2^{\circ}$ and $3^{\circ}$ per year. The wide range of inferred rotation rate is caused by the sensitivity of such studies to local complexities in structure which have been demonstrated to exist. Freeoscillation "splitting functions" are insensitive to local structure and are therefore better candidates for estimating differential IC rotation more accurately. We use a recently developed method for analyzing free oscillations which is insensitive to earthquake source, location and mechanism to constrain this differential rotation. In a prior study, we found that inner core differential rotation has been essentially zero over the last 20 years. We revisit this issue, including additional earthquakes and modes in our analysis. Our best estimate is a barely significant superrotation of $0.13 \pm 0.11^{\circ} \mathrm{yr}$, which is still consistent with the idea that the inner core is gravitationally locked to the mantle.
\end{abstract}

\section{Introduction}

The Earth's inner core enjoyed sudden public interest as "the planet within a planet" when seismologists found the first seismic evidence that it rotates faster than the mantle (Song and Richards, 1996; Su et al., 1996). The discovery was a timely one as some modern geodynamo simulations predicted this superrotation (Glatzmaier and Roberts, 1996), and earlier studies had already speculated that the inner core is likely rotating due to coupling through magnetic torques (e.g. Gubbins, 1981). Not all geodynamo calculations predict a superrotation though. For example, Kuang and Bloxham's (1997) dynamo calculations show that the inner core sometimes rotates faster and sometimes slower than the mantle which seems to be consistent with length-of-day observations (Buffett and Creager, 1999). The early seismic evidence came from the observation that differential body wave travel times between phases that turn deep in the outer core, $\operatorname{PKP}(\mathrm{BC})$, and phases that penetrate into the inner core, PKP(DF), change with time. Song and Richards (1996) observed a 0.3s change over 30 years for differential times measured for paths from a source region in the South Sandwich Islands (SSI) in the Atlantic Ocean to global seismic network (GSN) station COL (College, Alaska). Using certain assumptions about the structure of the inner core, this change in time was converted into a $1 \% \mathrm{yr}$ superrotation of the inner core. The assumptions involved are actually quite strict and turned out later to be too simplistic. Though the physical cause of inner core anisotropy is 
not yet well understood (see e.g. Jeanloz and Wenk, 1988; Karato, 1993; Yoshida et al., 1997; Bergman, 1997), it was assumed that the inner core behaves roughly like a single anisotropic crystal with a fast symmetry axis closely but not exactly aligned with the rotation axis of the Earth. The slight tilt of the symmetry axis which has been inferred by several groups (Su and Dziewonski, 1995; Shearer and Toy, 1991; McSweeney et al., 1997; Song, 1997) allows any differential rotation of the inner core to manifest itself in temporal variations of the travel times of body waves emanating from a fixed source region and recorded by a fixed receiver. The idea of the inner core behaving like a single crystal was quickly questioned when temporal variations were found for some paths (e.g. Song and Richards, 1996 for the path from the SSI source region to station COL) but not for others (e.g. Souriau, 1998a for the path from the Novaja Zemlya nuclear test site to GEOSCOPE station DRV, Dumont D'Urville in Antarctica). We now know that the path from SSI to COL is highly anomalous as it passes through regions of extremely heterogeneous structure having some of the largest gradients found so far (Creager, 1997). If unaccounted for, even simple large-scale heterogeneous structure can severely bias results for inner core rotation rates (Souriau, 1998b). Taking lateral heterogeneity into account Creager (1997) estimated the superrotation to be around $0.25 \% \mathrm{yr}$ which is significantly lower than initial estimates. Creager (2000) re-analyzed a large dataset of differential travel times and now sets the lower limit of the IC rotation rate to $0.15 \% \mathrm{yr}$ which is actually consistent with his observations of 1997 . This result would also be consistent with the small rate of $0.15 \% \mathrm{yr}$ found in a study that investigated the time-dependence of inner core scattered waves generated by sources at the Novaya Zemlya test site and observed at the LASA (Large Aperture Seismic Array) in Montana (Vidale et al., 2000). Song (2000) re-analyzed his dataset in a joint inversion for inner core structure and superrotation and now finds that the superrotation is $0.6 \% \mathrm{yr}$ which is less than $1 \% \mathrm{yr}$ but still the highest recently published value.

The process of measuring the time-dependence of the BC-DF differential times appears difficult for at least two reasons: 1) the data depend on the accurate location of earthquakes and it is certainly true that the locations of earlier events are less well known; 2) measuring the DF travel time for polar paths (e.g. the SSI-COL path) seems a difficult endeavor because the DF phase is often diffuse and quite small. A recent study suggests that the strategy of analyzing doublet events for which the waveforms are practically identical (Poupinet et al., 2000) instead of looking at the time-dependence of differential travel times is a more promising approach. Unfortunately, it seems that the definition of a doublet event is non-unique and the discovery of a time-dependent signal appears to be a matter of the analysis technique applied, the reference models used, and assumptions applied in regard of the polarity of the analyzed signals (Song, 2001; Poupinet and Souriau, 2001).

Another seismic dataset that is sensitive to inner core structure and hence has the potential of constraining inner core rotation is the normal mode dataset. It has long been known that compressional waves that travel parallel to the spin axis are significantly faster, arriving about 2 seconds earlier, than waves that travel in the equatorial 
plane (Poupinet et al., 1983). These observations eventually led to the discoveries described above. It has also been known, on the other hand, that free oscillations which sample the inner core are strongly split (Masters and Gilbert, 1981; see also Figure 1) by a structure which is dominantly axisymmetric and mimics the effect of an excess ellipticity of the Earth. Workers at Harvard (Woodhouse et al., 1986; Morelli et al., 1986) inferred that anisotropy of the inner core was the main reason for the anomalous observations in both mode splitting as well as body wave travel times. Normal modes provide a powerful tool to constrain differential inner core rotation that is, in certain ways, superior to the body wave method. Free oscillations are natural low-pass filters of 3D structure so long-wavelength phenomena, such as IC rotation, are prime study targets. Free oscillations "see" the Earth as a whole, so the observation of how a free oscillation splitting pattern changes with time and any inference on IC rotation is not biased by effects from localized structures. It is also not necessary to know the physical cause of these patterns (anisotropy or heterogeneity). All that needs to be observed is if they change with time.

The credit of being the first to study IC rotation with normal modes goes to Sharrock and Woodhouse (1998). They investigated the fit of "splitting functions" (see below) to the data of 5 inner-core sensitive modes under the assumption of a rotating inner core and inferred a westward rotation rate of 1 to more than $2^{\circ} / \mathrm{yr}$, which is obviously inconsistent with the body wave observations. We recently developed a new technique to analyze free oscillation splitting (Masters et al., 2000a) and re-examined inner-core sensitive modes for differential rotation. A convenient feature of this technique is that it is insensitive to errors in source location and mechanism. The analysis of nine modes resulted in a best-fitting IC rotation rate of $0.01 \pm 0.21 \% \mathrm{yr}$ eastward and we concluded that the inner core is most likely gravitationally locked to the mantle (Laske and Masters, 1999). The brevity of that paper did not allow us to discuss some of the details of the method or extend our discussion to subjects that are of obvious concern. For example, inner-core sensitive modes are also quite sensitive to mantle structure and it was suggested that our results depend on the "mantle correction" we are using to study the inner core (Creager, 2000). Here, we demonstrate in a synthetic test that our forward modelling strategy is capable of recovering an inner core rotation, if it exists. We also show that there is indeed some variability in the results when using different global mantle tomographic models but that the results are actually remarkably consistent. It turns out that inconsistencies in inferred rotation rates seem to be mode-specific rather than model-specific and we discuss possible causes for this. We also discuss cases for which our method cannot be applied. Foremost among these are strongly coupled modes and uncoupled modes that overlap in frequency with other modes of high harmonic degree.

\section{Spectra, Receiver Strips and Splitting Functions}

In this section, we briefly introduce the data and the essentials of mode seismology and summarize the autore- 
gressive method we use to analyze mode splitting. For details about the AR method the reader is referred to Masters et al. (2000a). The 1990ies have seen a renaissance in free oscillation seismology, not least because there were more than 25 extremely large earthquakes (Table 1) that were recorded on typically 100 observatory-quality broadband seismic instruments. All of these earthquakes excited numerous free oscillation overtones that are sensitive to inner core structure. Figure 1 shows typical examples of spectra for inner-core sensitive mode ${ }_{13} S_{2}$. Non-spherical structure splits the $2 \ell+1$ singlets of a $\operatorname{mode}_{n} S_{\ell}$. The effects of rotation and hydrostatic ellipticity of the Earth split the set of 5 singlets of ${ }_{13} \mathrm{~S}_{2}$ by $6.7 \mu \mathrm{Hz}$. When comparing the spectral lines at stations PPT (Papete, Tahiti) and SPA (South Pole) it becomes immediately clear that the actual splitting of this mode is much larger (more that $15 \mu \mathrm{Hz}$ ), i.e. this mode is anomalously split due to strong heterogeneity within the Earth. Because the shape of the spectra depends on the source-receiver geometry as well as the source mechanism, the peaks vary for different earthquakes at the same station (e.g. compare the spectra for stations ANMO or BFO). For each earthquake and each mode, we apply a trick to collapse the information contained in the roughly 100 time series (or spectra) into a set of only $2 \ell+1$ "receiver strips", without losing any information about 3D structure.

Our starting point is the representation of the time series of an isolated split multiplet at station $j$, first given by Woodhouse and Girnius (1982):

$$
u_{j}(t)=\sum_{k=1}^{2 \ell+1} R_{j k} a_{k}(t) e^{i \bar{\omega} t} \quad \text { or } \quad \mathbf{u}(t)=\mathbf{R} \cdot \mathbf{a}(t) e^{i \bar{\omega} t}
$$

where the real part is understood. The $j$ 'th row of $\mathbf{R}$ is a $2 \ell+1$ vector of spherical harmonics which describe the motion of the spherical-earth singlets at the $j$ 'th receiver and is readily calculated. $\bar{\omega}$ is the multiplet degenerate frequency and $\mathbf{a}(t)$ is a slowly varying function of time given by

$$
\mathbf{a}(t)=\exp (i \mathbf{H} t) \cdot \mathbf{a}(0)
$$

where $\mathbf{a}(0)$ is a $2 \ell+1$ vector of spherical-earth singlet excitation coefficients which can be computed if the source mechanism of the event is known. $\mathbf{H}$ is the "splitting matrix" of the multiplet and incorporates all the information about 3D structure, i.e.

$$
H_{m m^{\prime}}=\left(a+m b+m^{2} c\right) \delta_{m m^{\prime}}+\sum \gamma_{s}^{m m^{\prime}} c_{s}^{t}
$$

where $-\ell \leq m \leq \ell ;-\ell \leq m^{\prime} \leq \ell$ and $t=m-m^{\prime} . a, b$ and $c$ describe the effects of rotation and hydrostatic 
ellipticity (Dahlen, 1968), $\gamma_{s}^{m m^{\prime}}$ are integrals over three spherical harmonics which are easy to compute (e.g. Dahlen and Tromp, 1998) and the "structure coefficients", $c_{s}^{t}$, are given by

$$
c_{s}^{t}=\int_{0}^{a} \mathbf{M}_{s}(r) \cdot \delta \mathbf{m}_{s}^{t}(r) r^{2} d r
$$

$\delta \mathbf{m}_{s}^{t}$ are the expansion coefficients of the 3D aspherical Earth structure: $\delta \mathbf{m}(r, \theta, \phi)=\sum \delta \mathbf{m}_{s}^{t}(r) Y_{s}^{t}(\theta, \phi)$ and $\mathbf{M}_{s}$ are integral kernels which can be computed (Woodhouse and Dahlen, 1978; Woodhouse, 1980; Henson, 1989). Strictly speaking, equation 1 is not quite correct since both $\mathbf{R}$ and $\mathbf{a}(0)$ should include small renormalization terms (see Dahlen and Tromp, 1998, equations 14.87 and 14.88). Technically, we would need to know the splitting matrix before we can apply the renormalization which would require an iterative approach. However, for the (isolated) modes considered here, ignoring the renormalization terms leads to errors in $\mathbf{R}$ and $\mathbf{a}(0)$ on the order of a part in $10^{3}$ and does not affect our results in any significant way. The $Y_{s}^{t}=X_{s}^{t}(\theta) e^{i t \phi}$ is a spherical harmonic of harmonic degree $s$ and azimuthal order number $t$. An isolated mode of harmonic degree $\ell$ is sensitive to even-order structure only up to harmonic degree $s=2 \ell$. If the structure within the Earth is axisymmetric (e.g. rotation/ellipticity only), then the splitting matrix is diagonal, the individual singlets can be identified by the index $m$ and the only singlet visible at a station at the Earth's poles is the $m=0$ singlet.

Using equations (1) and (2) we now form the "receiver strips" for each event:

$$
\mathbf{b}(t)=\mathbf{R}^{-1} \cdot \mathbf{u}(t)=\exp [i(\mathbf{H}+\mathbf{I} \bar{\omega}) t] \cdot \mathbf{a}(0)
$$

We actually work in the frequency domain using spectra of Hanning-tapered records in a small frequency band about a multiplet of interest. Examples for the events of Figure 1 are given in Figure 2. The spectral lines in this diagram are proportional to the spectra of individual singlets, if axisymmetric structure dominates the splitting matrix. This is almost the case for modes which sample the inner core. Note that not every set of receiver strips exhibits a high signal-to-noise ratio. Depending on the source depth and mechanism, some of the largest earthquakes may very well not excite a specific mode particularly well and hence cannot be included in the analysis for this particular mode. This is the case for the Indian Ocean 2000 event for mode ${ }_{13} \mathrm{~S}_{2}$ (Figure 2), while on the other hand this earthquake produced high-quality strips for ${ }_{13} \mathrm{~S}_{3}$. For each event, we assign error bars to the receiver strips by performing a standard linear error propagation (e.g. Jackson, 1972) using the residual variance as a measure of data error.

We now use the autoregressive nature of the receiver strips to make our analysis technique independent of 
earthquake location and source mechanism. The receiver strips satisfy a recurrence in time (using equation 5):

$$
\mathbf{b}(t+\delta t)=\mathbf{R}^{-1} \cdot \mathbf{u}(t+\delta t)=\exp [i(\mathbf{H}+\mathbf{I} \bar{\omega})(t+\delta t)] \cdot \mathbf{a}(0)=\mathbf{P}(\delta t) \mathbf{b}(t)
$$

SO

$$
\begin{gathered}
\mathbf{b}(t+\delta t)=\mathbf{P}(\delta t) \mathbf{b}(t) \\
\text { where } \quad \mathbf{P}(\delta t)=\exp [i \delta t(\mathbf{H}+\mathbf{I} \bar{\omega})]
\end{gathered}
$$

and equation (6) has no term that depends on the seismic source. We can now set up an inverse problem for the propagator matrix $\mathbf{P}$, using the strips of all events simultaneously and then determine the splitting matrix $\mathbf{H}$ from $\mathbf{P}$ using the eigenvalue decomposition of $\mathbf{P}$ (Masters et al., 2000a). The matrix $\mathbf{H}$ we retrieve in this process is in general non-Hermitian. If we think of structure as having a real (elastic) and imaginary (anelastic) part, we can use the unique representation

$$
\mathbf{H}=\mathbf{E}+i \mathbf{A}
$$

where $\mathbf{E}=\frac{1}{2}\left(\mathbf{H}+\mathbf{H}^{H}\right)$ and $i \mathbf{A}=\frac{1}{2}\left(\mathbf{H}-\mathbf{H}^{H}\right)$ and superscript $H$ indicates Hermitian transpose. Both $\mathbf{E}$ and $\mathbf{A}$ are Hermitian and can be written:

$$
E_{m m^{\prime}}=\left(a+m b+m^{2}\right) \delta_{m m^{\prime}}+\sum_{s} \gamma_{s}^{m m^{\prime}} c_{s}^{t} ; \quad A_{m m^{\prime}}=\sum_{s} \gamma_{s}^{m m^{\prime}} d_{s}^{t}
$$

The $\gamma \mathrm{s}$ are the geometrical factors of equation (3), the $c_{s}^{t}$ are the elastic structure coefficients, and the $d_{s}^{t}$ are the anelastic structure coefficients. After removing the effects of the Earth's rotation and hydrostatic ellipticity, equations (8) can be regarded as a pair of linear inverse problems for $\mathbf{c}$ and $\mathbf{d}$ and we can explicitly include penalties for rough structure (i.e., high $s$ ) and so remove structure which is not required to fit the data. It is convenient to visualize the geographic distribution of structure as sensed by a mode by forming the elastic splitting function (Woodhouse and Giardini, 1985):

$$
f_{E}(\theta, \phi)=\sum_{s, t} c_{s}^{t} Y_{s}^{t}(\theta, \phi)
$$


and an equivalent function for anelastic structure where the $c_{s}^{t}$ are replaced by $d_{s}^{t}$.

Figure 3 shows an example of the procedure for mode ${ }_{13} \mathrm{~S}_{2}$. We use the receiver strips of 13 earthquakes that occurred between the 94 Fiji Islands and the 98 Molucca Sea events to determine the splitting matrix for this mode. After subtracting the effects of the Earth's rotation and hydrostatic ellipticity, each of the Hermitian matrices $\mathbf{E}$ and $\mathbf{A}$ are inverted for structure coefficients $c_{s}^{t}$ and $d_{s}^{t}$ which are then used to compute the splitting functions. The splitting functions are largely dominated by zonal structure $(s=0)$ but the smaller non-zonal components are very robustly determined, i.e. the patterns do not change significantly when varying the set of events or the frequency-band chosen to determine $\mathbf{H}$. We also observe that the anelastic signal is much smaller than the elastic signal (note that the scale for the elastic splitting function is twice that of the anelastic one). Unlike the elastic splitting functions, the anelastic ones also do not change coherently from mode to mode. This indicates that with the current set of earthquakes, we cannot yet determine anelastic structure reliably. We therefore will ignore the effects due to anelastic structure in the following sections.

\section{Looking for a Time-Dependent Signal}

\section{The Obvious Approach}

A straightforward approach to search for a time-dependent signal in the splitting functions is to determined the splitting matrices using only old events and only recent events and then compare the resulting elastic splitting functions. An example is shown in Figure 4 for mode ${ }_{13} \mathrm{~S}_{2}$. Using the 13 events described above, we determine the "recent" splitting function. The temporal distribution of the events gives us the splitting function at the time of the Flores Sea event (June 17, 1996). All 11 events between the 77 Tonga Islands and the 89 Macquarie Islands events are used to determine the "past" splitting function. The temporal distribution of these events gives the splitting function for August 18, 1982. An assumed differential inner core rotation rate of $1 \% \mathrm{yr}$ should let some of the patterns in the splitting function be out of phase by $14^{\circ}$. However, the splitting functions are remarkably similar and a phase shift of any pattern is not obvious (compare left panels in Figure 4a). A complicating factor in this comparison is the fact that inner-core sensitive modes are quite sensitive to structure of the Earth's mantle (e.g. Figure 6). To study the signal solely generated by inner core structure, the observed splitting functions should therefore be corrected for mantle signal which we assume does not change with time. To predict the mantle corrections we use our model SB10L18 (Masters et al., 2000b). We prefer this model over others because, as opposed to other models, mode data were included in the construction of this model and, perhaps equally important, bulk sound speed was determined independently of shear velocity. Bulk sound speed is negatively correlated with shear velocity at the base of the mantle, a region were anomalies are quite large. The negative 
correlation implies that perturbations in shear velocity change differently from those of compressional velocity. Using a shear-velocity model and standard scaling relationships between $\mathrm{V}_{s}$ and $\mathrm{V}_{p}$ to calculate the mantle predictions can potentially cause a bias in the splitting functions. The mantle correction for SB10L18 is shown is Figure 4a together with the "residual" splitting functions that now display only the contribution from the inner core (we assume a spherically symmetric outer core). The excellent agreement of the residual splitting functions (right panels in Figure 4a) does not suggest a relative rotation. To quantify our comparison, we determine the "best-fitting" rotation angle that give the highest correlation between the two maps, where only the non-zonal parts are considered in the calculations for the fit (including the zonal part always give a correlation well above 0.8). The best rotation to map the "past" splitting function into the "recent" one is a westward (!) rotation by about $7^{\circ}$, which is in concordance with the Sharrock and Woodhouse (1998) results but inconsistent with the body wave studies. A disturbing fact is that the components of different harmonic degree $s$ require different best-fitting rotation angles (i.e. degree 2 requires an eastward rotation while degree 4 requires a westward one). This is physically implausible if the inner core rotates as a rigid body. From this comparison one is left to conclude that either inner core rotation does not exist or that the splitting functions are not determined precisely enough to allow such a comparison. The latter is likely, especially for the "past" splitting functions for which far less seismograms are available than for the recent ones. Furthermore, harmonic degree 4 structure is probably less well determined than degree 2 structure because its spectral amplitudes are smaller. We therefore seek alternative ways to search for inner core rotation and a forward approach is described in the next section.

\section{A Forward Approach and a Synthetic Test}

A better way of testing for inner core rotation is by a hypothesis test that the inner core is differentially rotating about the rotation axis (Figure 5). We assume that the modern data accurately constrain the current splitting function of the mode and that we can properly correct for structure in the mantle. The corrected splitting function by assumption reflects only inner core structure which can now be rotated about the rotation axis using an assumed rotation rate. We then add the mantle contribution back in, construct a synthetic $\mathbf{H}$ from which we compute a synthetic $\mathbf{P}$ (note that we ignore the anelastic part in this test). This $\mathbf{P}$ is used in equation (6) to test if the assumed rotation rate provides a good fit to the receiver strips, $\mathbf{b}$, for a given event. For a given mode, all events have to give the same rotation rate. And for the hypothesis to be acceptable, ALL the modal splitting functions should appear to be rotating at the same rate. The data have to meet certain criteria to be considered in the hypothesis test which will be outlined in the next section. In this section we perform a synthetic test to convince ourselves that this forward approach is indeed capable of recovering an assumed differential rotation of the inner core. We perform this test for two inner-core sensitive modes, ${ }_{13} \mathrm{~S}_{2}$ and ${ }_{15} \mathrm{~S}_{3}$. The sensitivity of these modes to mantle structure is quite different, especially near the core-mantle boundary where the question of using different scaling 
relationships between $\mathrm{V}_{s}$ and $\mathrm{V}_{p}$ is important (Figure 6). We construct a model (Model 1) that is a 9-layer simplified version of our shear velocity model S16B30 (Masters et al., 1996) and a simple model of inner core structure that has a large $c_{2}^{0}$ component and smaller $c_{2}^{2}$ and $c_{4}^{3}$ components. The contributions of synthetic mantle and inner core to the splitting functions are illustrated in Figure 7. To demonstrate how severely the mantle contribution masks an assumed inner-core rotation rate in the approach discussed in the last section, we create a second set of splitting functions for which the inner core is rotated by $20^{\circ}$. We then seek the best-fitting rotation angle that brings the two splitting functions for ${ }_{13} S_{2}$ in phase. As can be seem from Figure 8, the rotation angle is largely underestimated using the complete splitting functions, so an accurate mantle correction is indeed essential for that approach. To test the forward approach laid out in this section, we calculate synthetic seismograms for our model using the coupled-mode code of Park and Gilbert (1986). Only self-coupling and 1D attenuation is considered but the Earth's rotation and hydrostatic ellipticity is included in the calculations and the same steps are performed in this test as with real data. Using equation (5), we construct the receiver strips for both modes and two earthquakes (the 94 Bolivia and the 96 Flores Sea events) from the synthetics (Figure 9). Note that not all singlets are excited equally well by the events (the figure shows true spectral amplitudes, while Figure 2 shows normalized ones). The results from the hypothesis test are shown in Figure 10. Using equation (6) we determine the misfit for the receiver strips for both events and modes, assuming inner core rotation angles between -90 and $90^{\circ}$. The minimum in the misfit curves are at the expected rotation angle $\left(+20^{\circ}\right)$, to within $1^{\circ}$. Slight changes in the mantle model for the correction do not alter the outcome of this test significantly. For example, model 2 is S16B30 used in the original parameterization (30 natural B-splines radially) and CRUST5.1 (Mooney et al., 1998) added near the surface (though the crustal contribution to these modes is quite small). We find that the rotation angle is not recovered reliably only in cases when the mantle model is changed quite significantly. Model 3 is SB10L18 and the prediction for this model can be quite different from that of S16B30 for certain modes (e.g. compare those for ${ }_{13} \mathrm{~S}_{2}$ of Figures 4 and 7). The angles can be both under- as well as over-estimated though the example shown underpredicts the angle by 4 to $10^{\circ}$. We should point out that the success of our hypothesis test depends on the structure of the inner core. If the signal were 5 times smaller, or the structure was a pure $c_{2}^{2}$ term then our method could not reliably recover a given rotation angle. The observations in the next section will show that the signal caused by structure in the inner core is not as small and not as simple as this, so the results of our synthetic test are more pessimistic than we should expect for the real Earth.

\section{Splitting Functions for Real Data}

We have analyzed a suite of 15 inner-core sensitive modes and determine the "recent" splitting functions which are shown in Figure 11. The sensitivity of these modes to structure within the Earth can be estimates from inspecting the energy densities in Figure 6. Note that most splitting functions are largely dominated by degree 
2 zonal structure, though this is somewhat masked for high-frequency modes that are increasingly sensitive to mantle structure (e.g. compare Figures 11 and 12 for mode ${ }_{23} S_{5}$ ). There are some exceptions to this (e.g. ${ }_{9} S_{3}$ and, to a much lesser extent, ${ }_{3} S_{2}$ ) and we suspect that these splitting functions are less well determined and should probably not be included in our analysis (see later section for possible causes for this). The splitting functions for modes of low harmonic degree are quite simple because, as mentioned above, an isolated mode of degree $\ell$ is sensitive only to structure of degree up to $s=2 \ell$. It turns out that such modes are dominated even more by zonal structure after they have been corrected for mantle signal (Figure 13). This dominance is so strong for $\ell=1$ modes that it hampers a reliable determination of inner core rotation angles and we therefore do not include these in our analysis. Typical misfit curves for the hypothesis test for mode ${ }_{13} S_{2}$ as those obtained for the synthetic test (Figure 10) are shown in Figure 14. Note that the trough of the misfit function is narrower than in the synthetic experiment suggesting that the structure in the synthetic experiment was too simple and that rotating structure in the real inner core can be traced more reliably. We have shown earlier that some events excite a specific mode better than others so the receiver strips with a poor signal-to-noise ratio have to be identified and discarded. We devise a series of tests that the receiver strips have to pass. The most obvious criterion is that the fit to the receiver strips with any non-zero inner core rotation must be better than that using no rotation. Receiver strips with small initial misfit indicate that the event either did not excite this mode particularly well or that the mode couples to other modes and are so discarded. The reconstructed splitting matrix has to give a variance reduction greater than $70 \%$ and the receiver strips should be fit close to their error bars (the variance reduction is typically more than 90\%). A large residual misfit or a small variance reduction may indicate either a large anelastic contribution to the splitting matrix or strong coupling with another mode, both of which is ignored here. A non-zero rotation angle also has to yield a better fit to the data than the zonal structure alone (horizontal grey lines in Figure 14). In a few cases, different mantle corrections yield significantly different rotation angles. We regard this as an indicator of serious noise contamination and discard these events, for a particular mode. Figure 14 shows three examples for events that fail the tests for mode ${ }_{13} S_{2}$. All three events fail the test because the strips are noisy and the initial misfit is too small. In addition to this, the variance reduction of two of them is also too small $(8514,7101)$, one has no obvious minimum for a reasonable rotation angle (7101) and for one the improvement of fit to the strips by the non-zonal component is insignificant (7256).

\section{Determining the Final Rotation Rates}

The final two steps to determine the best-fitting differential inner core rotation rate are quite straight forward. In the first step, we determine the rotation rate for each mode. This is done by fitting a straight line to the rotation angles for all events. In the second step, we average the results over all modes. The first step is the crucial one because the assignment of error bars effectively weighs the results of different events. This process is somewhat 
subjective as the forward modelling process has no formal error propagation and we examine 3 different ways. High signal-to-noise events typically yield small residual misfit as well as a large relative difference between the fit given by the total splitting function vs. the zonal part only (i.e. grey line vs. bottom of the trough in Figure 14). We take the residual misfit and the relative trough depth as two possible error bars, $\sigma_{R M}$ and $\sigma_{T D}$. The typical halfwidth of a trough in the misfit function (the half width of the trough below the grey line) is about $25^{\circ}$ so we scale both errors by $12.5^{\circ}$, which we regard as conservative choice. For the third type of error, we simply assume uniform errors for all events, i.e. average scaled $\sigma_{T D}$ over all events and re-assign the average to all events. All three sets of errors yield about the same rotation rates, where the residual misfit error, $\sigma_{R M}$, exhibits a slightly larger scatter in the rotation rates for different modes. We take this as indicative that the exact choice of error bars is less significant than we had originally anticipated. Our preferred error bars are the $\sigma_{T D}$ because they yield the most consistent results. Figure 15 shows the rotation angles as function of event date for the nine modes for which more than 20 events passed the tests mentioned above. Some modes are marginally consistent with an eastward inner core rotation rate of $1 \% \mathrm{yr}\left(\right.$ e.g. $\left.{ }_{23} \mathrm{~S}_{5}\right)$ but others are clearly not (e.g. $\left.{ }_{10} \mathrm{~S}_{2},{ }_{18} \mathrm{~S}_{4}\right)$, and all modes give rotation rates smaller than $0.67^{\circ} \mathrm{yr}$. The rotation rates for all modes are summarized in Figure 16 and the average over all modes, the final differential inner core rotation rate is $0.13 \pm 0.11^{\circ} / \mathrm{yr}$. This rate is somewhat higher than the $0.01 \pm 0.21 \%$ yr given in (Laske and Masters, 1999) but is within their error bars. Our result is also consistent with recent body wave studies that give eastward rotation rates between 0.15 and $0.25^{\circ} / \mathrm{yr}$ (e.g. Creager, 2000; Vidale et al., 2000).

\section{Does the Rotation Rate depend on the Mantle Correction?}

An inspection of Figure 6 and the comparison of the splitting functions for the mantle corrections in Figures 4 and 7 suggest that the final inner core rotation could depend on the mantle correction we apply. We therefore repeated our analysis for a variety of mantle models that have recently been published. These include our older shear-velocity model S16B30 (Masters et al., 1996), our recent high-resolution model SB4L18 (Masters et al., 2000b), the recent Harvard model S362D1 (Gu and Dziewonski, 1999) and the recent Berkeley $\mathrm{V}_{S H}$ model SAW24B16 (Megnin and Romanowicz, 2000). We also calculate mantle corrections for the recent Caltech model using both their shear and compressional velocity models S20RTS and P20RTS (Ritsema and van Heist, 2000). Figure 17 shows the misfit curves for mode ${ }_{13} S_{3}$ for the 94 Bolivia event. The misfit functions are strikingly similar for the different mantle models and yield the same rotation angles to within $3^{\circ}$ of our estimate using model SB10L18. The Bolivia event has a high signal-to-noise ratio but we want to stress that the great similarity between misfit functions is rather typical and not an exception. We take this as indicative that current different mantle models give the same inner core rotation rates. That this is indeed the case is further stressed by the results shown in Figure 16. Almost all rotation rates obtained for different mantle models lie within the error 
bars of the rates obtained with SB10L18, our preferred mantle model. In fact, variations in rotation rate seem to depend on the mode, not on the mantle correction. For example, almost all models give westward rotation rates for modes ${ }_{3} \mathrm{~S}_{2},{ }_{10} \mathrm{~S}_{2},{ }_{13} \mathrm{~S}_{2}$ and ${ }_{16} \mathrm{~S}_{5}$. With one exception the final average rotation rates using the different models lie within the error bars of the SB10L18-value $\left(0.13 \pm 0.11 \% \mathrm{yr}\right.$ ). The rates are: $0.07 \% \mathrm{yr}$ (SB4L18), $0.08^{\circ} / \mathrm{yr}$ (S/P20RTS), $0.22 \% \mathrm{yr}$ (SAW24B16), $-0.05 \% \mathrm{yr}$ (S362D1) and 0.01\% $\mathrm{yr}$ (S16B30). The variation in these values

indicates that the mantle correction does influence the final rotation rate somewhat. It is rather obvious however that rotation rates outside of the range of roughly -0.05 to $0.25 \% \mathrm{yr}$ are clearly inconsistent with our mode data. Our preferred values are those obtained with joint S-/P- models (SB10L18 and S/P20RTS) as these take into account the anomalous relative behavior of shear and compressional velocity anomalies at the base of the mantle. The two values $(0.13$ and 0.08$)$ are in striking agreement.

\section{Discussion}

Early on in our analysis, we found that $\ell=1$ modes can constrain inner core rotation only poorly because the mantle-corrected splitting functions are dominated by a large zonal component. We can identify two more types of modes that, at this point, cannot be used in our analysis. The first type of modes are not coupled to other modes (or only weakly coupled) but coincide in frequency with other modes of relatively high $\ell$. One such mode is ${ }_{2} \mathrm{~S}_{3}$ which has considerable shear-energy in the inner core so would be ideal to study inner core rotation. Its degenerate frequency in the PREM model is $1.24219 \mathrm{mHz}$ (Dziewonski and Anderson, 1981). Immediately adjacent to it are ${ }_{0} \mathrm{~T}_{7}(1.22070 \mathrm{mHz}),{ }_{0} \mathrm{~S}_{7}(1.23179 \mathrm{mHz})$ and ${ }_{1} \mathrm{~T}_{1}(1.23611 \mathrm{mHz})$ and these modes can potentially couple (see also Deuss and Woodhouse, 2001). According to the selection rules for coupling modes, none of these modes couple through rotation or ellipticity but limited coupling can occur through 3D structure. In this mode group and for realistic Earth models, the mode pairs that couple strongest are ${ }_{0} S_{7}$ with $T_{7} T_{7}$ and ${ }_{0} S_{7}$ with ${ }_{1} T_{1}$, while the coupling between the $l=7$ modes and ${ }_{2} \mathrm{~S}_{3}$ exists but is extremely weak. Even without significant coupling, ${ }_{0} \mathrm{~S}_{7}$ is close enough in frequency to ${ }_{2} S_{3}$ that the receiver strips of ${ }_{0} S_{7}$ and ${ }_{2} S_{3}$ need to be computed simultaneously. If the coupling between ${ }_{2} S_{3}$ and ${ }_{0} S_{7}$ were negligible, we could then consider only the strips for ${ }_{2} S_{3}$ and perform our hypothesis test. For a given mode pair, we need at least $2 \times\left(l^{\prime}+l+1\right)$ records per event to do this, which gives 22 records for the mode pair ${ }_{0} \mathrm{~S}_{7-2} \mathrm{~S}_{3}$. Some records for the events listed in Table 1 are not suitable for a particular mode because of insufficient data volume (e.g. when an instrument stopped recording singificantly earlier than Q-cycles of the mode), so that the hypothesis test cannot be performed for ${ }_{2} \mathrm{~S}_{3}$ for events prior to 1986. For obvious reasons, this is not desirable and we therefore exclude modes like ${ }_{2} \mathrm{~S}_{3}$ from our analysis.

Another group of modes are strongly coupled modes. As with weakly coupled modes, we would include both modes to compute the receiver strips. But in this case, the strips of a mode can no longer be treated as those for 
an isolated mode and the complete splitting matrix, including the cross-coupling blocks have to be determined. This fact complicates the hypothesis test immensely as all four blocks (the two self-coupling blocks of the two modes and the two cross-coupling blocks) need to be decomposed and re-assembled using assumed rotation rates. We therefore do not include such modes in our study. This is particularly unfortunate, since this affects some of the $\ell=2$ modes which are particularly sensitive to inner core structure but sometimes strongly coupled to radial modes. An example is the pair ${ }_{7} S_{2-}{ }_{2} S_{0}$ (Masters et al., 2000c). ${ }_{7} S_{2}$ has a considerable amount of shear-energy at the top of the inner core (Figure 6) and so carries invaluable information about the inner core. However, the coupling to ${ }_{2} S_{0}$ is strong enough to inhibit a clear identification of the $m=0$-line of ${ }_{7} S_{2}$. Even if we treat ${ }_{7} S_{2-2} S_{0}$ as coupled modes, the splitting function we retrieve from the self-coupling block of ${ }_{7} S_{2}$ does not have the expected shape of a dominant $c_{2}^{0}$ component with large positive local frequency shifts being at the poles. We rather observe negative frequency shifts at the poles (similar to the splitting function of ${ }_{9} \mathrm{~S}_{3}$ in Figure 11). Synthetic tests with coupled-mode seismograms using simple realistic structures for the inner core cannot reproduce this observation so this phenomenon is currently not understood. Deuss and Woodhouse (2001) find significant distortions of individual synthetic spectra of mode ${ }_{13} S_{2}$ when coupling this mode with ${ }_{5} S_{0}$. The frequency shifts due to coupling are predicted to be rather small for these two modes, possibly because the modes are separated in frequency by almost $40 \mu \mathrm{Hz}$. We notice though that the cross-coupling blocks can get quite large (e.g. coupling through the Earth's ellipticity). We do not observe a similar distortion of the receiver strips of ${ }_{13} S_{2}$ as we see for ${ }_{7} S_{2}$ so we expect the bias in the splitting functions introduced by ignoring coupling to be rather small. We notice however that both ${ }_{13} S_{2}$ and ${ }_{10} S_{2}$ (which is equally weakly coupled to ${ }_{4} S_{0}$ ) give westward rotation for the inner core. A westward rotation is also seen for ${ }_{3} S_{2}$ the coupling properties of which are quite complex (see also Zürn et al., 2000). It is not obvious why inner core rotation rates for these modes should systematically be biased westward, but for the sake of argument we could assume that this is the case and eliminate their results. Even when not taking into account the rates for the $\ell=2$-modes, the final inner core rotation rate is as small as $0.34 \pm 0.13 \% \mathrm{yr}$ eastward which is inconsistent with the rotation rate of $0.6 \% \mathrm{yr}$, a recent result from Song's re-iterated body wave data (Song, 2000) and we can rule out any greater rate with confidence.

A caveat when analyzing modes using the isolated-mode assumption is that only even degree structure can be determined. It is known from body wave studies that the heterogeneity at the top of the inner core has a strong $s=1$ signal that is roughly divided into a western and an eastern hemisphere (Tanaka and Hamaguchi, 1997; Creager, 2000). The fact that isolated modes are insensitive to such structure does not affect the ability of a mode analysis to track down the differential rotation, provided the inner core rotates as a rigid body. Structure of uneven harmonic degree can potentially be determined by analyzing coupled modes. For a coupling mode pair (same type only, i.e. S-S, T-T) to be sensitive to $s=1$ structure the selection rules predict that the harmonic degree of the modes need to be different by an odd number. The analysis of the coupling blocks for modes ${ }_{13} S_{2}$ and ${ }_{9} S_{7}$ 
can therefore potentially recover $s=1$ structure of the inner core, though the coupling between these modes by our Model 1 (mantle and simple inner core) appears to be relatively weak. Further treatment of this will be the subject of a future contribution.

In summary, we believe a modal analysis is the best way to determine inner core rotation. First, we are dealing with large-scale vibrations which are insensitive to errors in event locations and to local structure in the inner core. Second, the method is independent of the earthquake source mechanism. Third, we do not need to worry about how much of the splitting functions we observe are caused by heterogeneity or anisotropy - all we care about is whether they change with time. After obtaining an initial estimate of $0.01 \pm 0.21 \% \mathrm{yr}$ we have augmented our study with data from additional earthquakes. With the analysis of a larger suite of modes we now obtain a small superrotation of $0.13 \pm 0.11 \%$ yr. This result is still marginally consistent with the small rotation rates reported in most recent body wave analyses but rules out any rates significantly beyond $0.35 \%$ yr. Our current best value indicates that the inner core is super-rotating at a barely significant rate relative to the mantle. This is in accord with the notion that the inner core is gravitationally locked to the mantle (Buffett and Creager, 1999) in which case the inner core could exhibit small time-dependent differential rotation rates.

\section{Acknowledgments}

The data used in this study were collected at a variety of global and regional seismic networks (IRIS-USGS, IRIS-IDA, GEOSCOPE, TERRAscope, BDSN, GEOFON) and obtained from the IRIS-DMC, GEOSCOPE, NCEDC, GEOFON and BFO. We thank Ken Creager, Ruedi Widmer-Schnidrig and Jeroen Tromp for discussion and helpful reviews. This research has been supported by National Science Foundation grants EAR98-09706 and EAR00-00920. 


\section{References}

Bergman, M.I., Measurements of elastic anisotropy due to solidification texturing and the implications for the Earth's inner core. Nature, 389, 60-63, 1997.

Buffett, B.A., and K.C. Creager, A comparison of geodetic and seismic estimates of inner-core rotation. Geophys. Res. Let., 26, 1509-1512, 1999.

Creager, K.C., Inner core rotation rate from small-scale heterogeneity and time-varying travel times. Science, 278, 1248-1288, 1997.

Creager, K.C., Inner Core Anisotropy and Rotation. In: Earth's Deep Interior: Mineral Physics and Tomography, AGU Monograph, 117, eds Karato et al, Washington DC, pp.89-114, 2000.

Dahlen, F.A., The normal modes of a rotating, elliptical earth. Geophys. J. R. Astron. Soc., 16, 329-367, 1968.

Dahlen, F.A., and J. Tromp, Theoretical Global Seismology. Princeton University Press, 1998.

Deuss, A., and J.H. Woodhouse, Theoretical free-oscillation spectra: the importance of wide band coupling. Geophys. J. Int., 146, 833-842, 2001.

Dziewonski, A.M., and D.L. Anderson, Preliminary reference Earth model. Phys. Earth Planet. Inter., 25, 297-356, 1981.

Glatzmaier, G.A., and P.H. Roberts, Rotation and Magnetism of Earth's Inner Core. Science, 274, 1887-1891, 1996.

Gu, Y.J., and A.M. Dziewonski, Mantle Discontinuities and 3-D Tomographic Models. EOS Trans. AGU, 80, F717, 1999

Gubbins, D., Rotation of the Inner Core. J. Geophys. Res., 85, 11,695-11,699, 1981.

Henson, I.H., Multiplet coupling of the normal modes of an elliptical, transversely isotropic Earth. Geophys. J. Int., 98, 457-459, 1989.

Jackson, D.D., Interpretation of inaccurate, insufficient and inconsistent data. Geophys. J. R. Astron. Soc., 28, 97-109, 1972.

Jeanloz, R., and H.-R. Wenk, Convection and anisotropy of the inner core. Geophys. Res. Lett., 15, 72-75, 1988.

Karato, S., Inner Core Anisotropy Due to the Magnetic Field-Induced Preferred Orientation of Iron. Science, 262, 1708-1711, 1993.

Kuang, W., and J. Bloxham, An Earth-like Numerical dynamo model. Nature, 389, 371-374, 1997.

Laske, G., and G. Masters, Limits on the Rotation of the Inner Core from a new Analysis of Free Oscillations. Nature, 402, 66-68, 1999.

Masters, G., and F. Gilbert, Structure of the inner core inferred from observations of its spheroidal shear modes. Geophys. Res. Lett., 8, 569-571, 1981.

Masters, G., S. Johnson, G. Laske, and H. Bolton, A shear velocity model of the mantle. Phil. Trans. R. Soc. Lond., 354A, 1385-1411, 1996.

Masters, G., G. Laske, and F. Gilbert, Autoregressive estimation of the splitting matrix of free oscillation multiplets. Geophys. J. Int, 141, 25-42, 2000a.

Masters, G., G. Laske, and H. Bolton. A. Dziewonski, The relative behavior of shear velocity, bulk sound speed, and compressional velocity in the mantle: implications for chemical and thermal structure. In: Earth's Deep Interior: Mineral Physics and Tomography, AGU Monograph, 117, eds Karato et al, Washington DC, pp.63-87, 2000b.

Masters, G., G. Laske, and F. Gilbert, Matrix autoregressive analysis of free-oscillation coupling and splitting. Geophys. J. Int, 143, 478-489, 2000c. 
McSweeney, T.J., K.C. Creager, and R.T. Merrill, Depth extent of inner core seismic anisotropy and implications for geomagnetism. Phys, Earth. Planet. Int., 101, 131-156, 1997.

Megnin, C., and B. Romanowicz, The 3D shear velocity of the mantle from the inversion of body, surface, and higher mode waveforms. Goephys. J. Int., 143, 709-728, 2000.

Mooney, W.D., G. Laske, and G. Masters, CRUST 5.1: A global crustal model at $5^{\circ}$ X $5^{\circ}$. J. Geophys. Res., 103, 727-747, 1998.

Morelli, A., A.M. Dziewonski, and J.H. Woodhouse, Anisotropy of the inner core inferred from PKIKP travel times. Geophys. Res. Lett., 13, 1545-1548, 1986.

Park, J., and F. Gilbert, Coupled free oscillations of an aspherical dissipative rotating earth: Galerkin theory. $J$. Geophys. Res., 91, 7241-7260, 1986.

Poupinet, G., R. Pillet, and A. Souriau, Possible heterogeneity of the earth's core deduced from PKIKP travel times. Nature, 305, 204-206, 1983.

Poupinet, G., A. Souriau, and O. Coutant, The extistence of an inner core super-rotation questioned by teleseismic doublets. Phys. Earth Planet. Int., 118, 77-88, 2000.

Poupinet, G., and A. Souriau, Reply to Xiadong Song's comment on "The existence of an inner core super-rotation questioned by teleseismic doublet”. Phys. Earth Planet. Int., 124, 275-279, 2001.

Ritsema, J., and H. Van Heijst, Seismic imaging of structural heterogeneity in Earth's mantle: evidence for large-scale mantle flow. Science Progress, 83, 243-259, 2000.

Sharrock, D.S., and J.H. Woodhouse, Investigation of time dependent inner core structure by the analysis of free oscillation spectra. Earth, Planets, and Space, 50, 1013-1018, 1998.

Shearer, P.M., and K.M. Toy, $P K P(B C)$ veresus $P K P(D F)$ differential travel times and aspherical structure in Earth's inner core. J. Geophys.Res., 96, 2233-2247, 1991.

Song, X., and P.G. Richards, Seismological evidence for differential rotation of the Earth's inner core. Nature, 382, 221-224, 1996.

Song, X., Anisotropy of the Earth's inner core. Reviews of Geophys., 35, 297-313, 1997.

Song, X., Joint inversion for inner core rotation, inner core anisotropy, and mantle heterogeneity. J. Geophys. Res., 105, 7931-7943, 2000.

Song, X., Comment on "The existence of an inner core super-rotation questioned by teleseismic doublets" by Georges Poupinet, Annie Souriau, and Oliver Coutant. Phys. Earth Planet. Int., 124, 269-273, 2001.

Souriau, A., New seismological constraints on differential rotation rates of the inner core from Novaya Zemlya events recorded at DRV, Antarctica. Geophys. J. Int., 134, F1-5, 1998a.

Souriau, A., Earth's inner core - is the rotation real?. Science, 281, 55-56, $1998 \mathrm{~b}$.

Su, W-J., and A.M. Dziewonski, Inner core anisotropy in three dimensions. J. Geophys. Res., 100, 9831-9852, 1995.

Su, W., A.M. Dziewonski, and R. Jeanloz, Planet within a planet: rotation of the inner core of the Earth. Science, 274, 1883-1887, 1996.

Tanaka, S., and H. Hamaguchi, Degree one heterogeneity and hemispherical variation of anisotropy in the inner core from $P K P(B C)-P K P(D F)$ times. J. Geophys. Res, 102, 2925-2938, 1997.

Vidale, J.E., D.A. Dodge, and P.S. Earle, Slow differential rotation of the Earth's inner core indicated by temporal changes in scattering. Nature, 405, 445-448, 2000.

Woodhouse, J.H., The coupling and attenuation of nearly resonant multiplets in the earth's free oscillation spectrum. Geophys. J. R. Astron. Soc., 61, 261-283, 1980.

Woodhouse, J.H., and F.A. Dahlen, The effect of a general aspherical perturbation on the free oscillations of the earth. Geophys. J. R. Astron. Soc., 53, 335-354, 1978. 
Woodhouse, J.H., and D. Giardini, Inversion for the splitting function of isolated low order normal mode multiplets. EOS Trans. AGU, 66, 300, 1985.

Woodhouse, J.H., D. Giardini, and X.-D. Li, Evidence for inner core anisotropy from free oscillations. Geophys. Res. Lett., 13, 1549-1552, 1986.

Woodhouse, J.H., and T.P. Girnius, Surface waves and free oscillations in a regionalized earth model. Geophys. J. R. Astron. Soc., 68, 653-673, 1982.

Yoshida, S., I. Sumita, and M. Kumazagawa, Growth model of the inner core coupled with the outer core dynamics and the resulting elastic anisotropy. J. Geophys. Res., 101, 28085-28103, 1997.

Zürn, W., G. Laske, R. Widmer-Schnidrig, and F. Gilbert, Observation of Coriolis coupled modes below $1 \mathrm{mHz}$. Geophys. J. Int., 143, 113-118, 2000. 


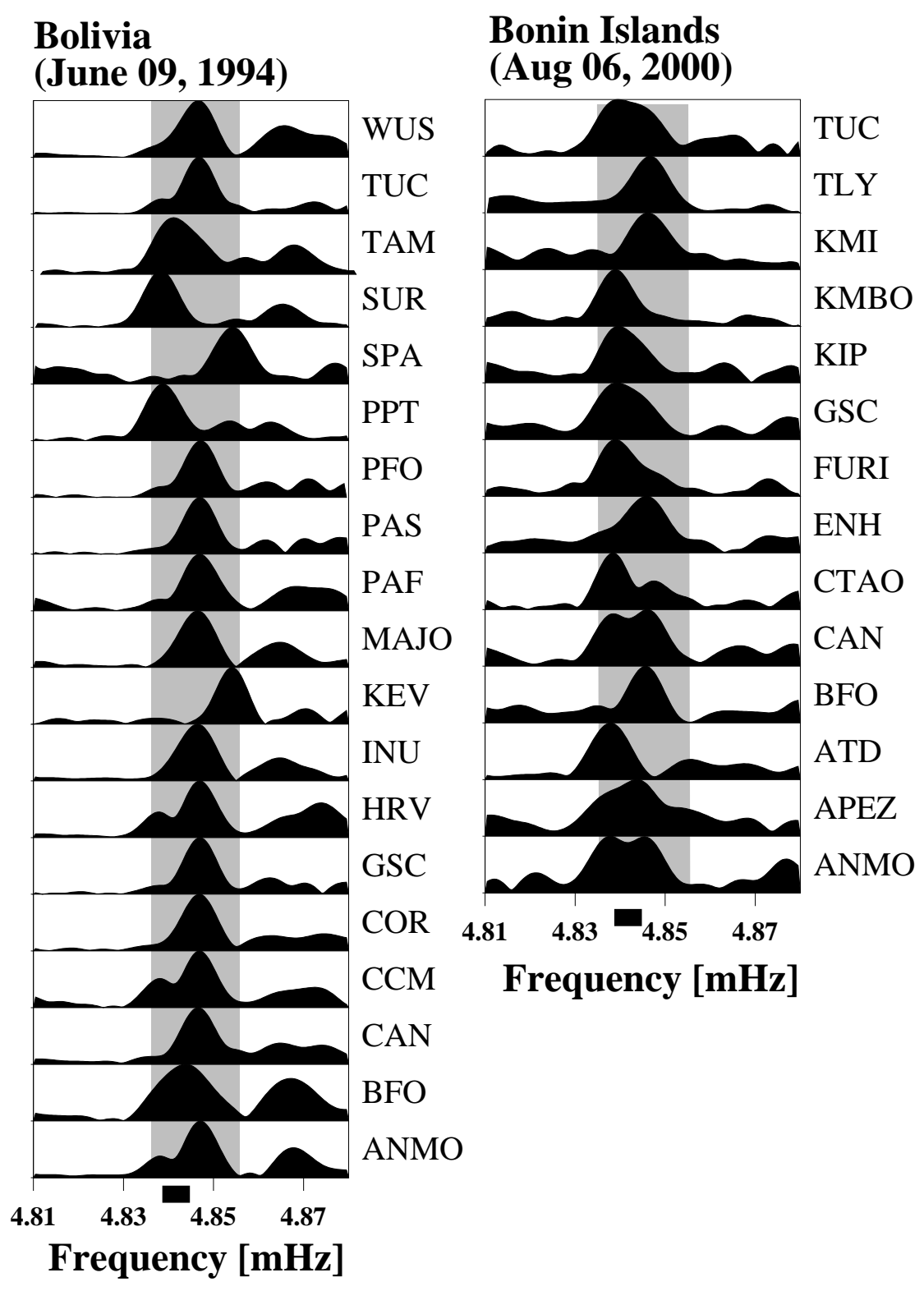

Figure 1. Hanning-tapered spectra of anomalously split inner-core sensitive mode ${ }_{13} S_{2}$ from vertical component recordings of the Bolivian (June 09, 1994) and the Bonin Islands (August 06, 2000) earthquakes. The spectra are taken at stations of various global and regional seismic broad-band networks (GEOSCOPE, IRIS-IDA, IRISUSGS, GEOFON and TERRAScope), starting $5 \mathrm{~h}$ after the event and using $65 \mathrm{~h}$ long records. Due to 3D structure of the Earth, the "spectral peak" exhibits fine-scale splitting, within a band defined as the splitting width (grey area). Some spectra even exhibit clearly split peaks (ANMO, CCM). Rotation and hydrostatic ellipticity of the Earth cause a splitting width of only $6.7 \mu \mathrm{Hz}$ (black bar). The smaller peaks at $4.87 \mathrm{mHz}$ are the faster decaying mode ${ }_{9} \mathrm{~S}_{7}$. 

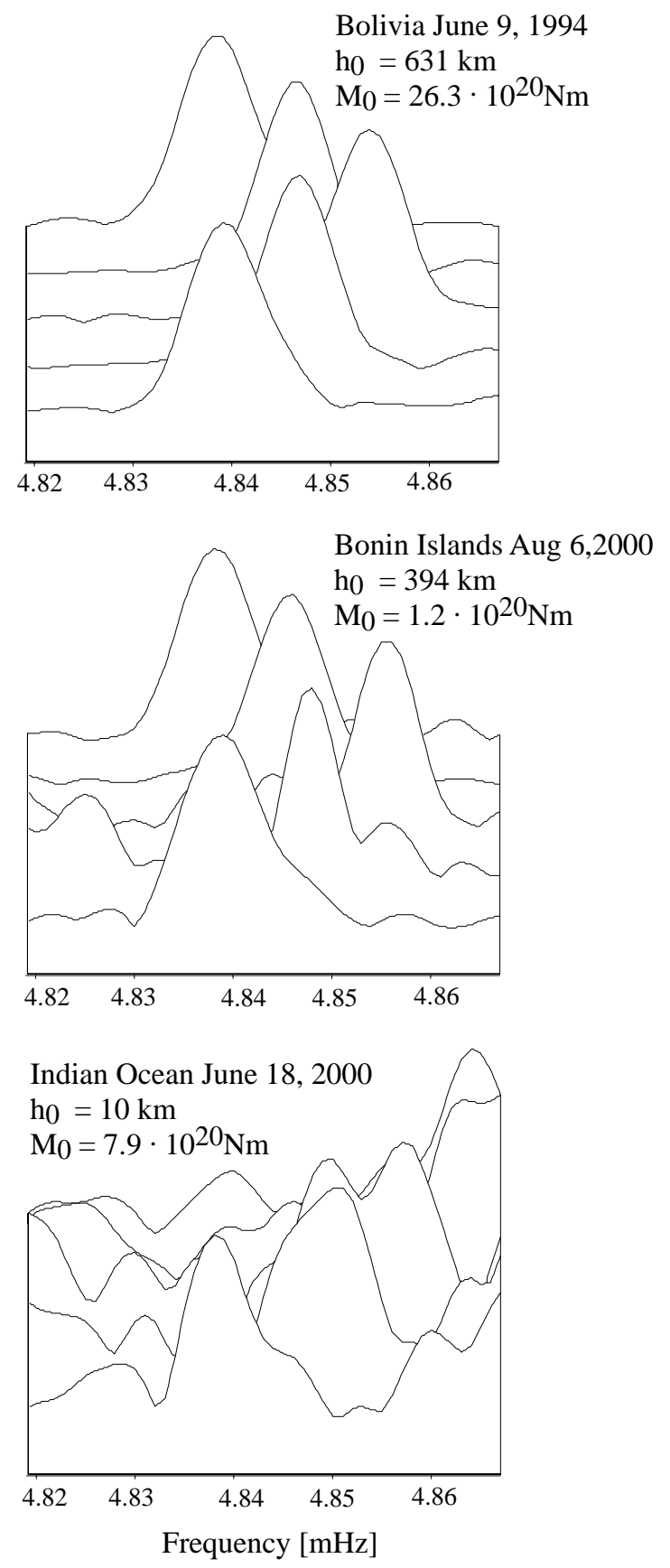

Figure 2. Receiver strips for mode ${ }_{13} S_{2}$ for the Bolivia and the Bonin Islands events. Even though the latter was 20 times smaller, it excited the mode well enough to produce high signal-to-noise strips. On the other hand, the greater Indian Ocean event on June 19, 2000 did not excite this particular mode sufficiently well to be considered in the analysis of inner core rotation. 


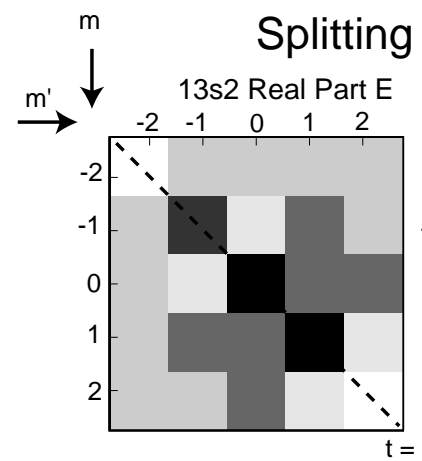

Matrix

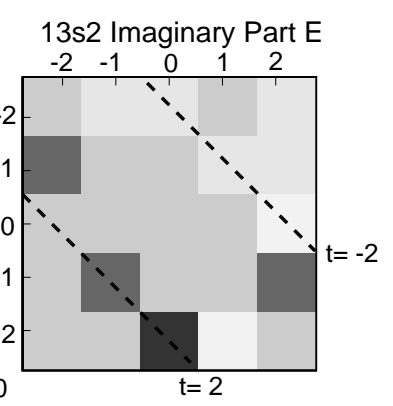

Splitting Function
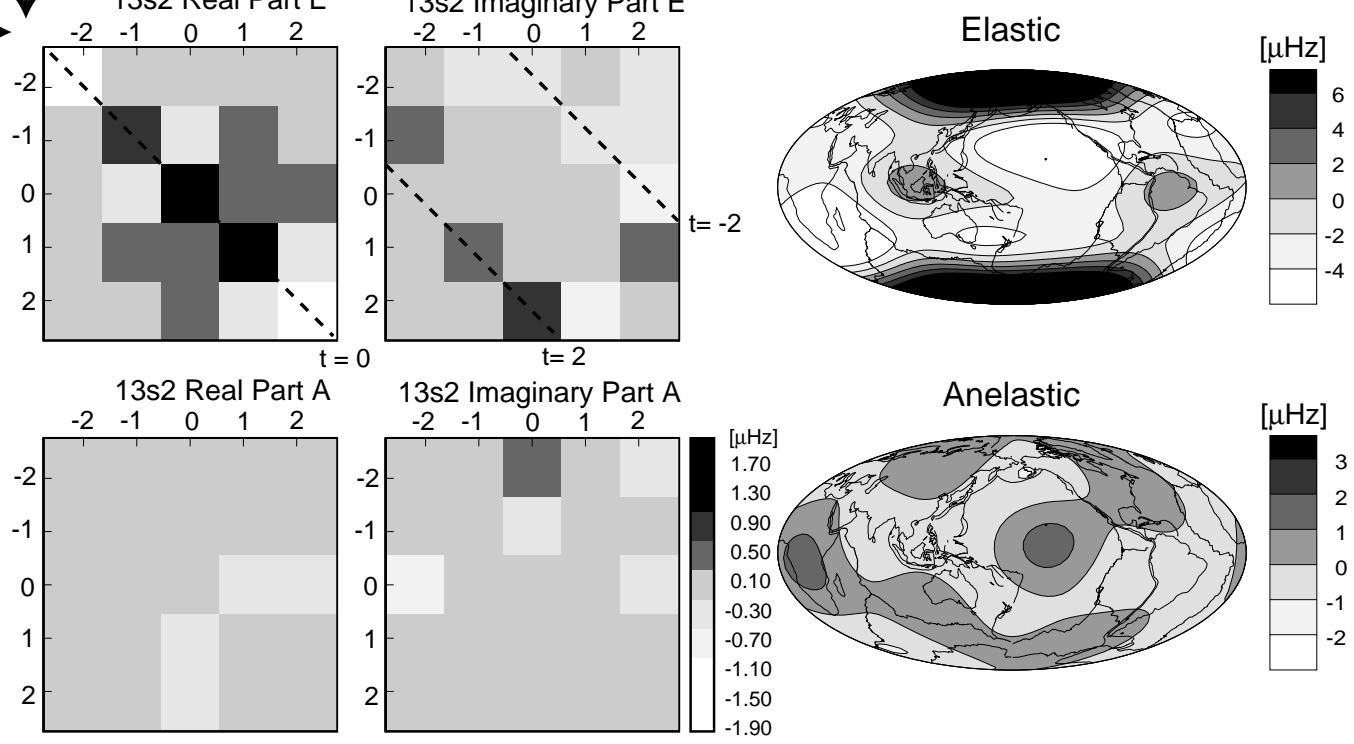

Figure 3. Left: Observed complete splitting matrix for mode ${ }_{13} S_{2}$, decomposed into its elastic (E) and anelastic (A) parts. Receiver strips of 13 earthquakes between the 94 Fiji Islands and the 98 Molucca Sea events entered the inversion. The signal down the diagonal of the matrices is caused by zonal structure $(t=0)$, as constrained by the selection rules for a mode. Also indicated are the contributions from $t= \pm 2$-structure (which is sectoral for $s=2$ ). Right: Splitting functions obtained from the splitting matrix on the left. The signal from anelastic structure is typically much smaller than that from elastic structure. 


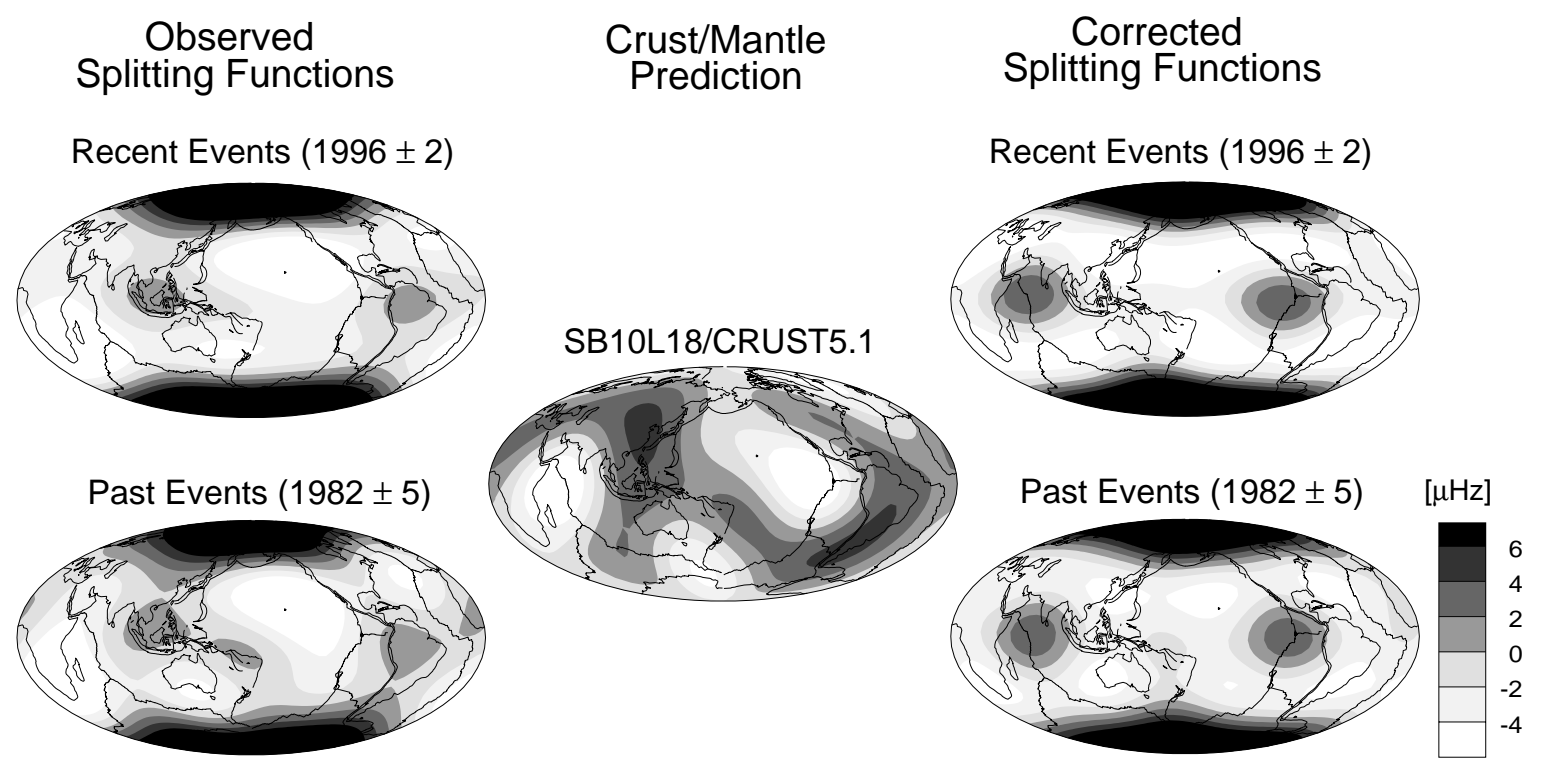

Figure 4a. Left: Observed splitting functions for mode ${ }_{13} S_{2}$, using 13 recent events (94 Fiji Islands up to 98 Molucca Sea) and using 11 past events (77 Tonga Islands up to 89 Macquarie Islands). Middle: The prediction for the contribution to the splitting function from crustal (CRUST 5.1 of Mooney et al. 1998) and mantle structure (SB10L18 of Masters et al. 2000b). Right: Splitting function after subtracting the crustal and mantle signal. The remaining signal must come from structure in the core, most likely the inner core. The two splitting functions are extremely similar and no obvious shift between them is visible. 


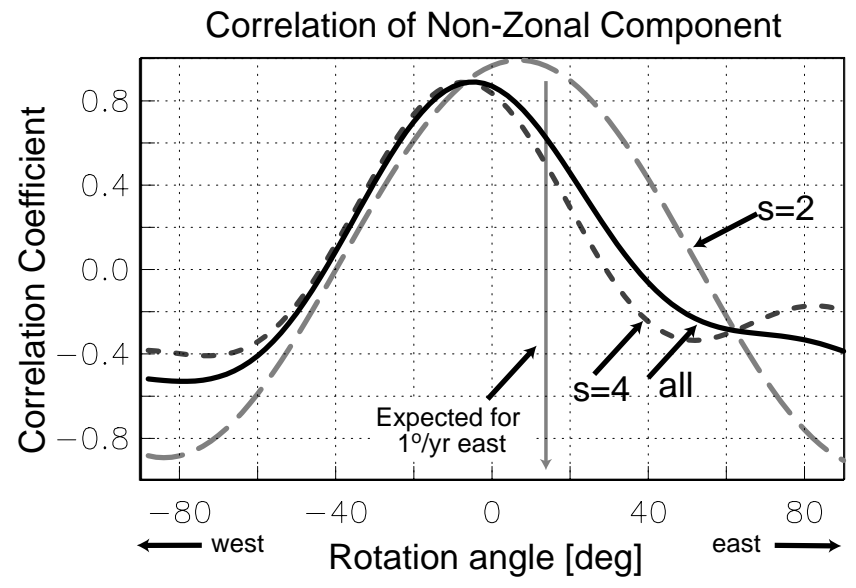

Figure 4b. Correlation between the two "core" splitting functions of Figure 4a, as function of rotation angle for the "past" splitting function. Only the non-zonal component is considered. The dominant zonal component forces the correlation to be above 0.8 , independent of the rotation angle. Harmonic degrees 2 and 4 require different angles for the highest correlation which is inconsistent with the inner core rotating as a rigid body. 


\section{Hypothesis Test}

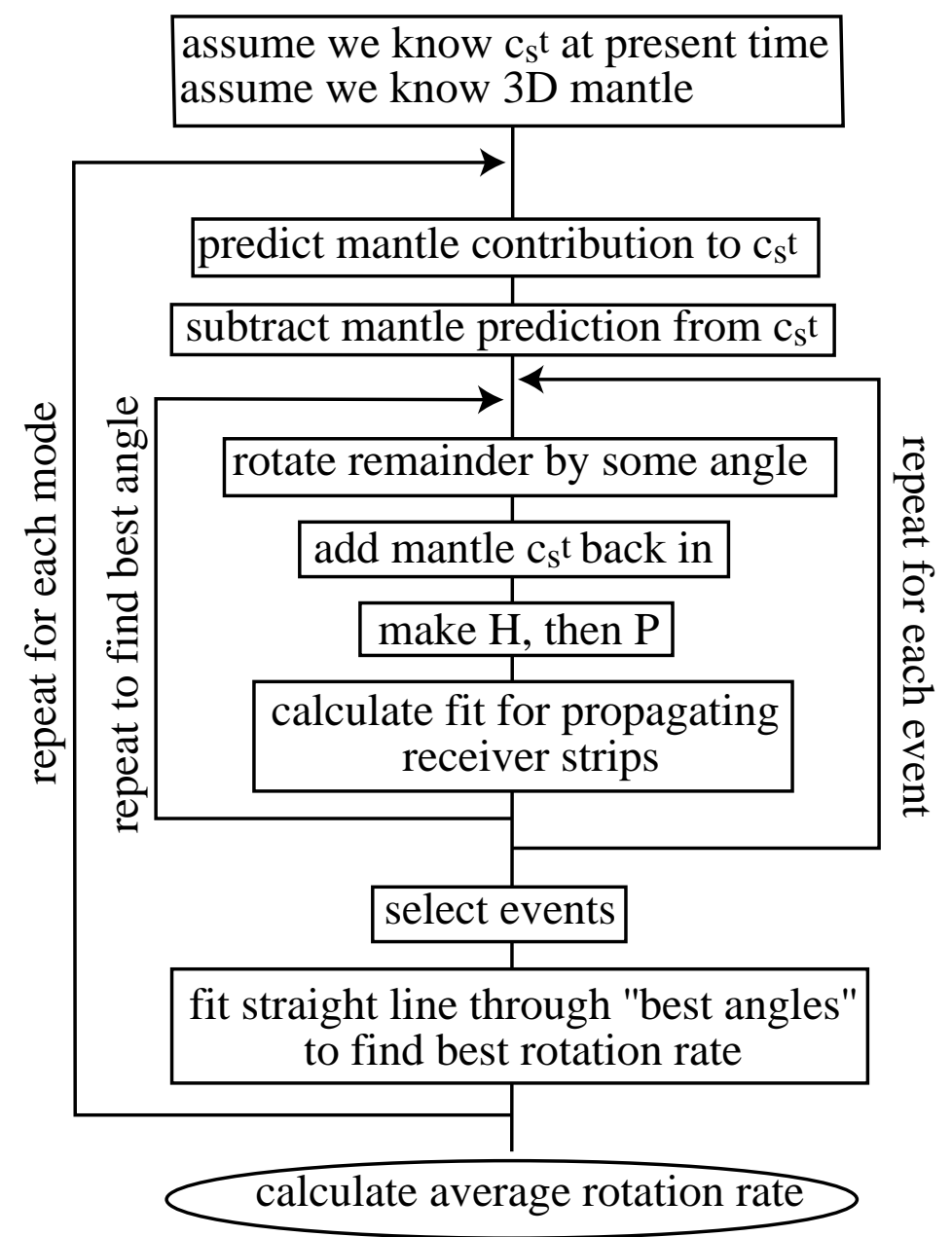

Figure 5. Flow chart for the hypothesis test for differential inner core rotation. 


\section{Energy Densities for the Modes used in this Study}
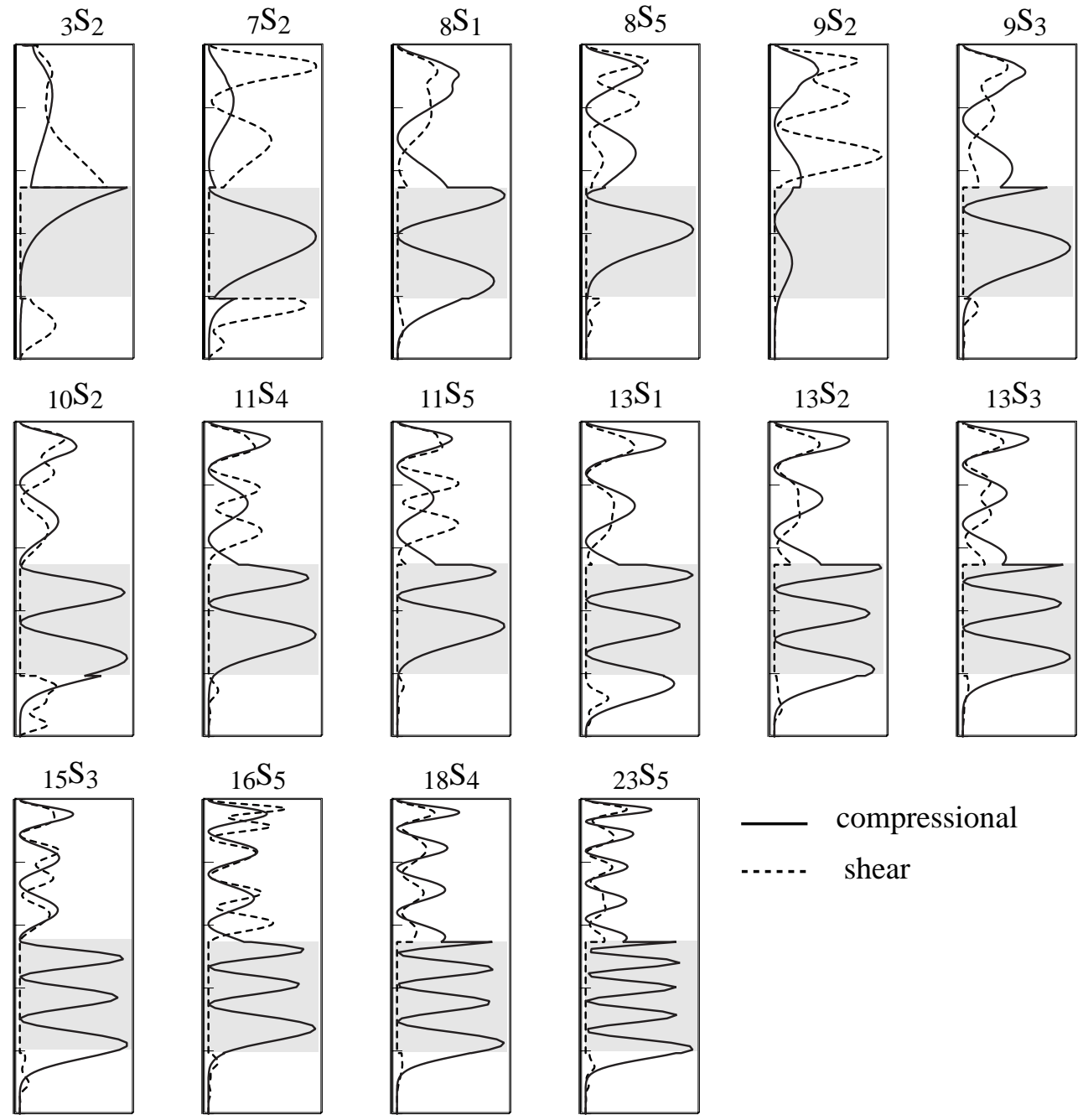

_ compressional

..... shear

Figure 6. Energy densities for a 1D Earth model for compression and shear as function of radius for the modes in this study. The sensitivity to structure in a 3D Earth slightly varies laterally which is taken into account in the calculation of the splitting functions. The grey area marks the outer core where the shear energy density is zero. 

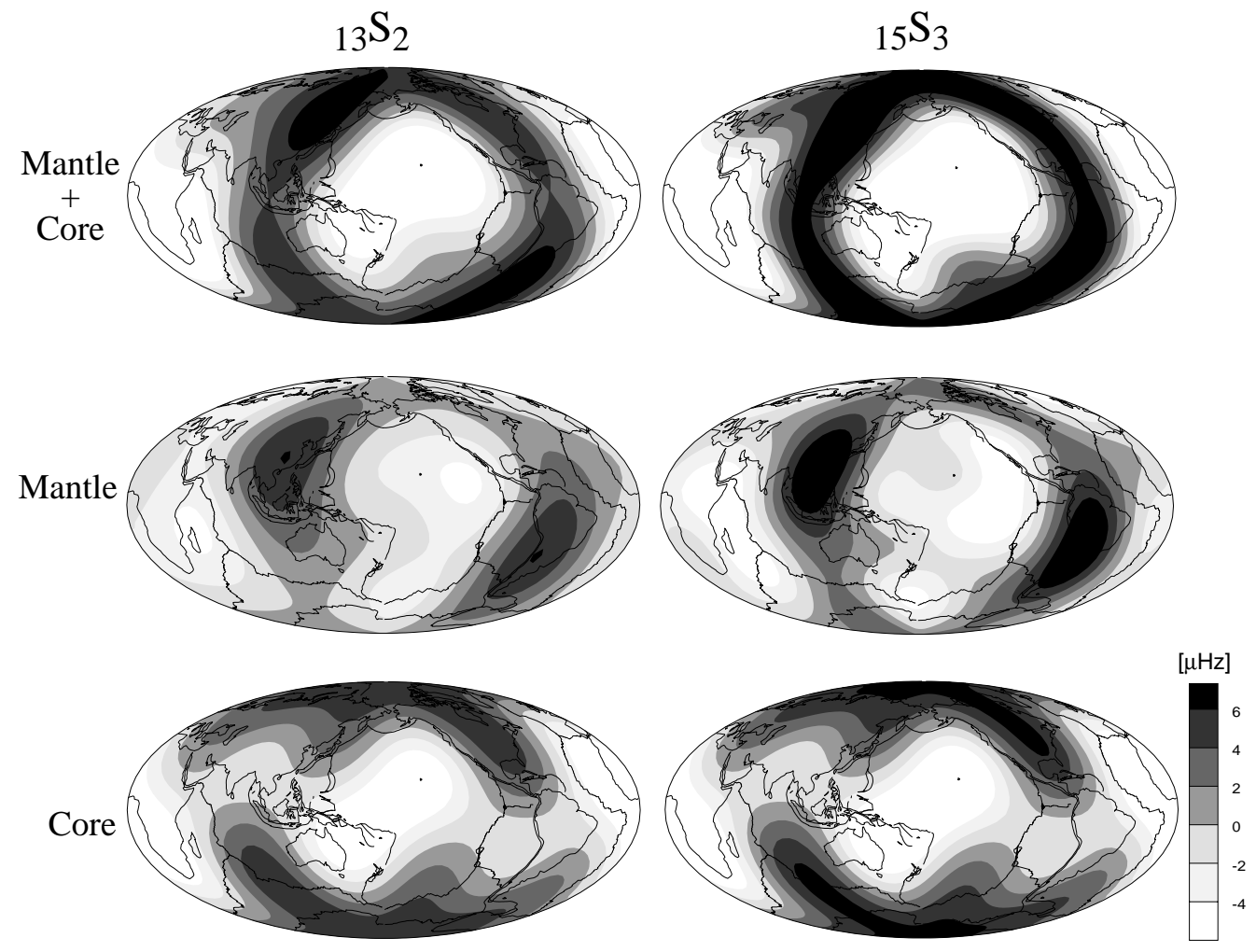

Figure 7. Splitting functions for the synthetic test for modes ${ }_{13} S_{2}$ and ${ }_{15} S_{3}$. The "mantle" is a simplified version of S16B30 (Masters et al., 1996). The "core" signal comes from a 200km thick layer at the top of the inner core (contributions from $c_{2}^{0}, c_{2}^{2}$, and $c_{4}^{3}$ ). 

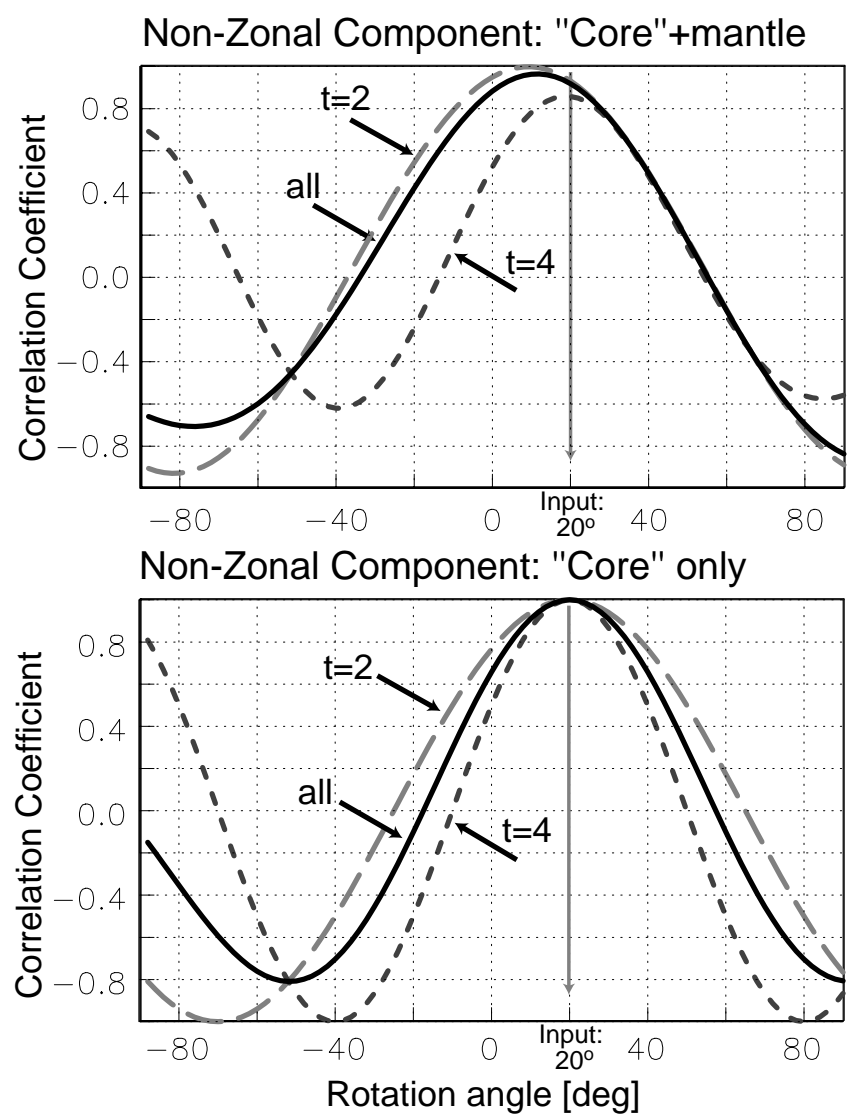

Figure 8. Correlation between two synthetic splitting functions for which the "core" part was rotated by $20^{\circ}$. Only the non-zonal component is considered. Note that the rotation angle is recovered only after the mantle contribution has been subtracted. 

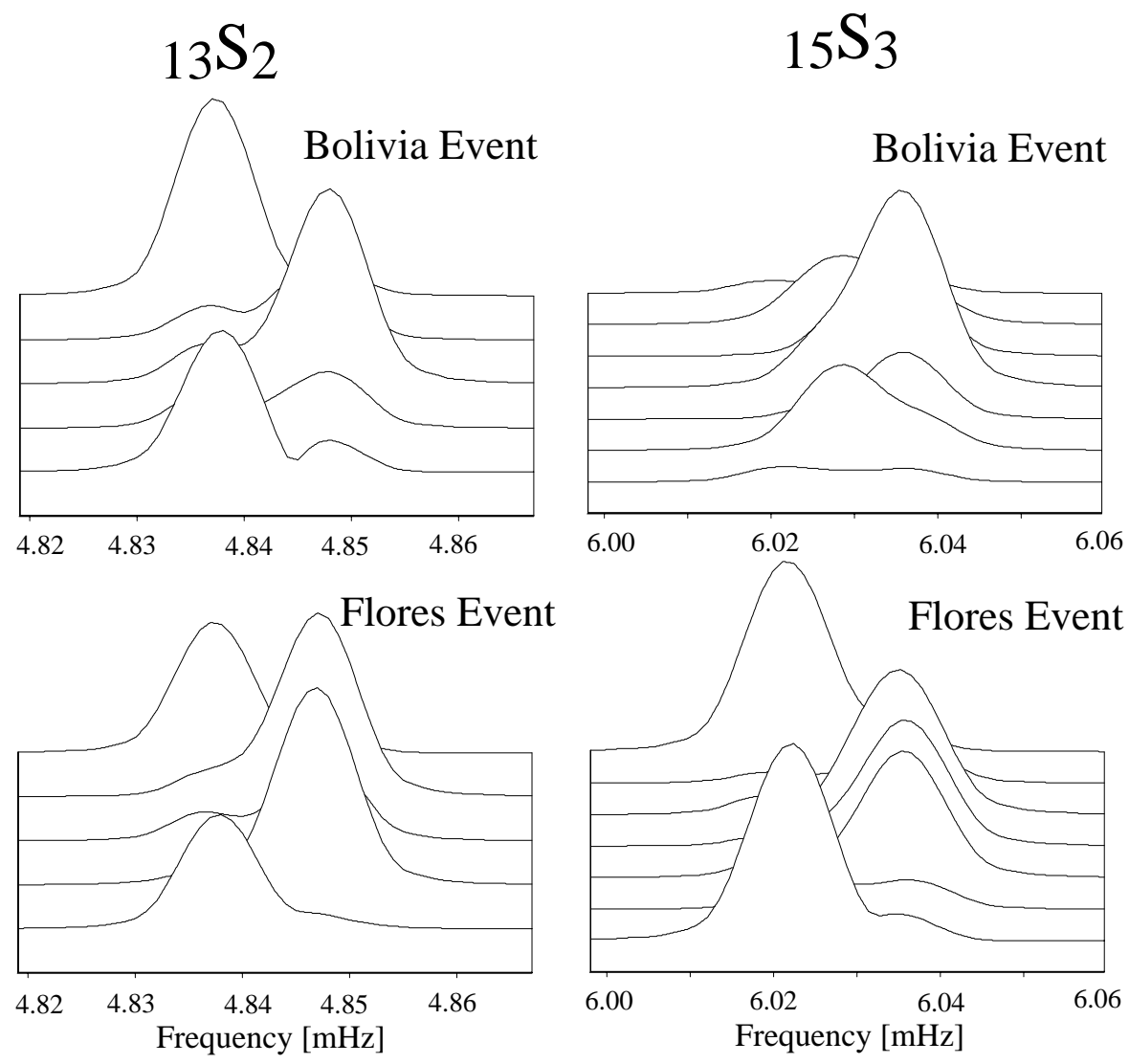

Figure 9. Receiver strips for modes ${ }_{13} S_{2}$ and ${ }_{15} S_{3}$ made from coupled-mode synthetic seismograms (self-coupling only) for the 94 Bolivia and 96 Flores Sea events. 

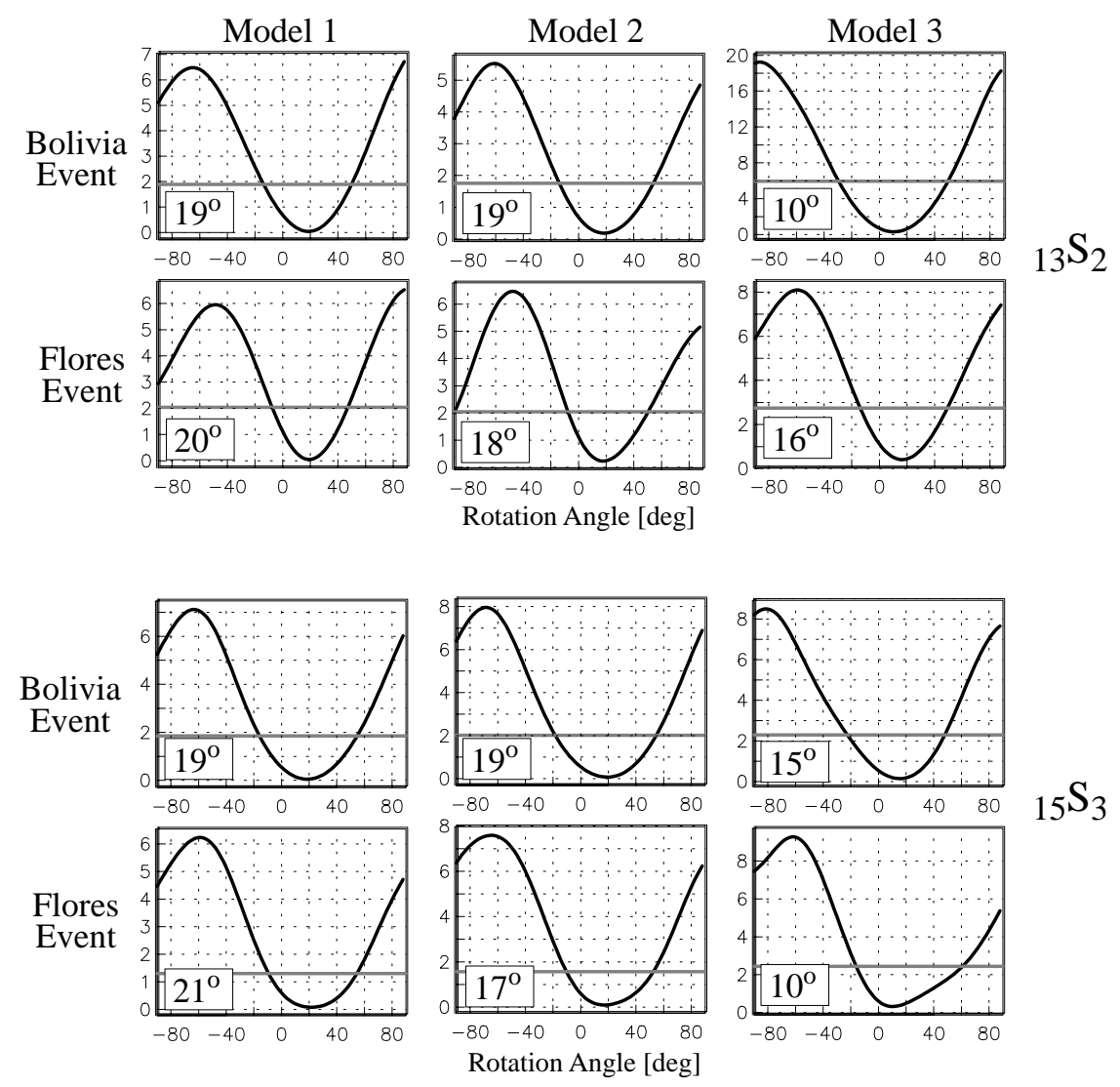

${ }_{15} \mathrm{~S}_{3}$

Figure 10. Misfit functions for the two modes and events resulting from the hypothesis tests using three different mantle models for the correction. The rotation angle corresponding to the minimum misfit is marked in the lower left corner. Models 1 and 2 usually give the right angle to within a degree, while model 3 can give a significantly smaller angle. The horizontal grey line marks the fit for the zonal component. 


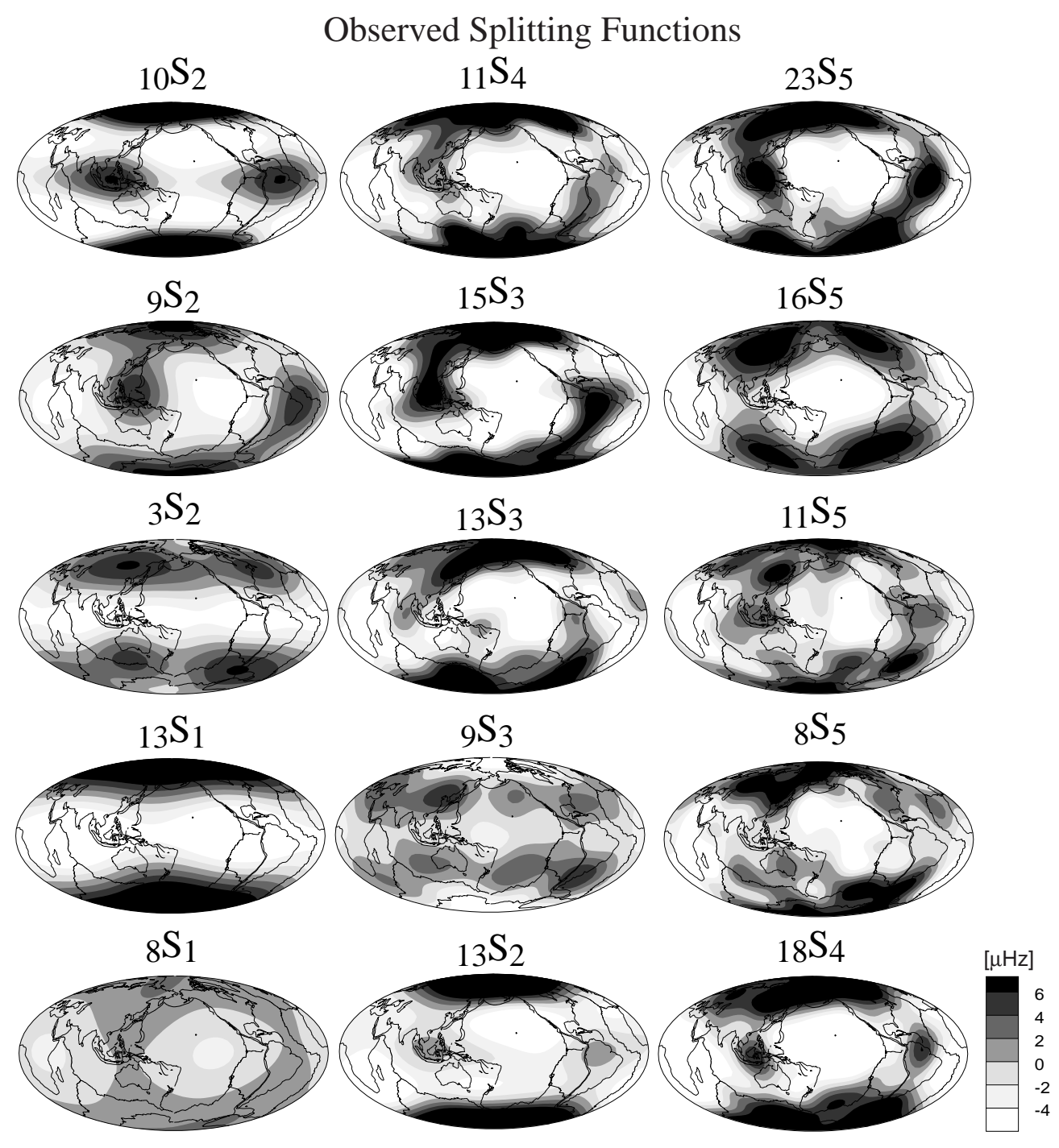

Figure 11. Splitting functions for the 15 inner-core sensitive modes analyzed in this study. The result for mode ${ }_{9} S_{3}$ is somewhat uncertain. (The effects due to rotation and hydrostatic ellipticity have been removed). 


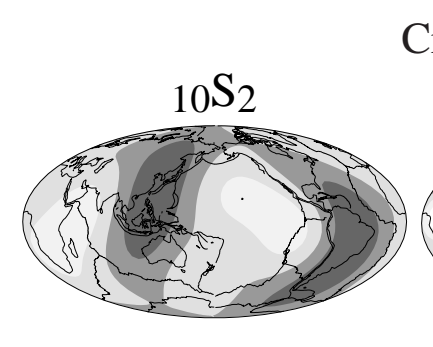

Crust+Mantle Predictions
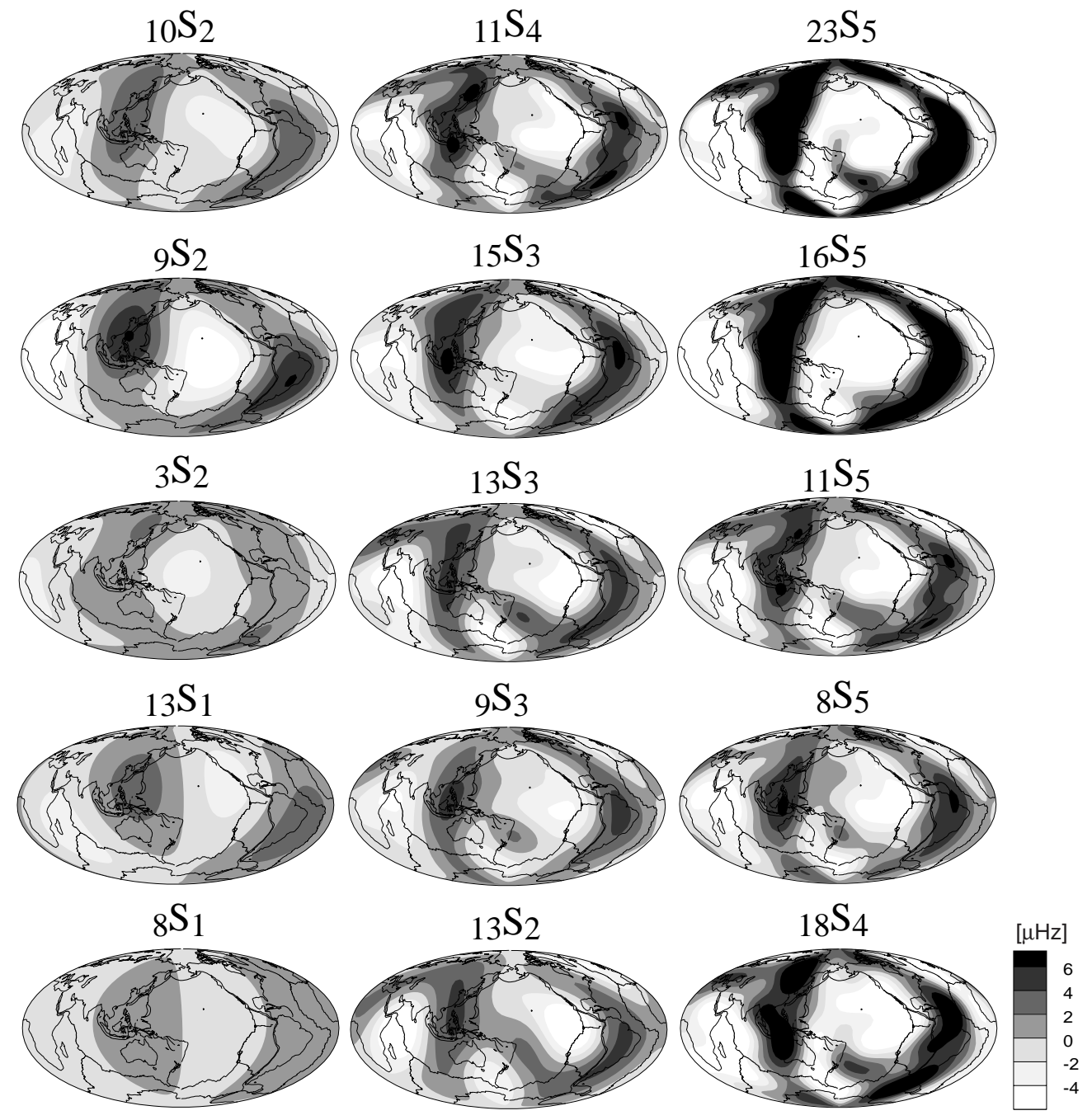

Figure 12. Contributions to the splitting functions of Figure 11 from crustal (Crust 5.1, Mooney et al., 1998) and mantle (SB10L18, Masters et al., 2000b) structure. The signal is usually moderate (and smaller than the observed signal) but is significant for high-frequency modes ${ }_{16} S_{5}(6.83 \mathrm{mHz}),{ }_{18} S_{4}(7.24 \mathrm{mHz})$ and ${ }_{23} S_{5}(9.29$ $\mathrm{mHz}$ ) which sense the shallow mantle. 


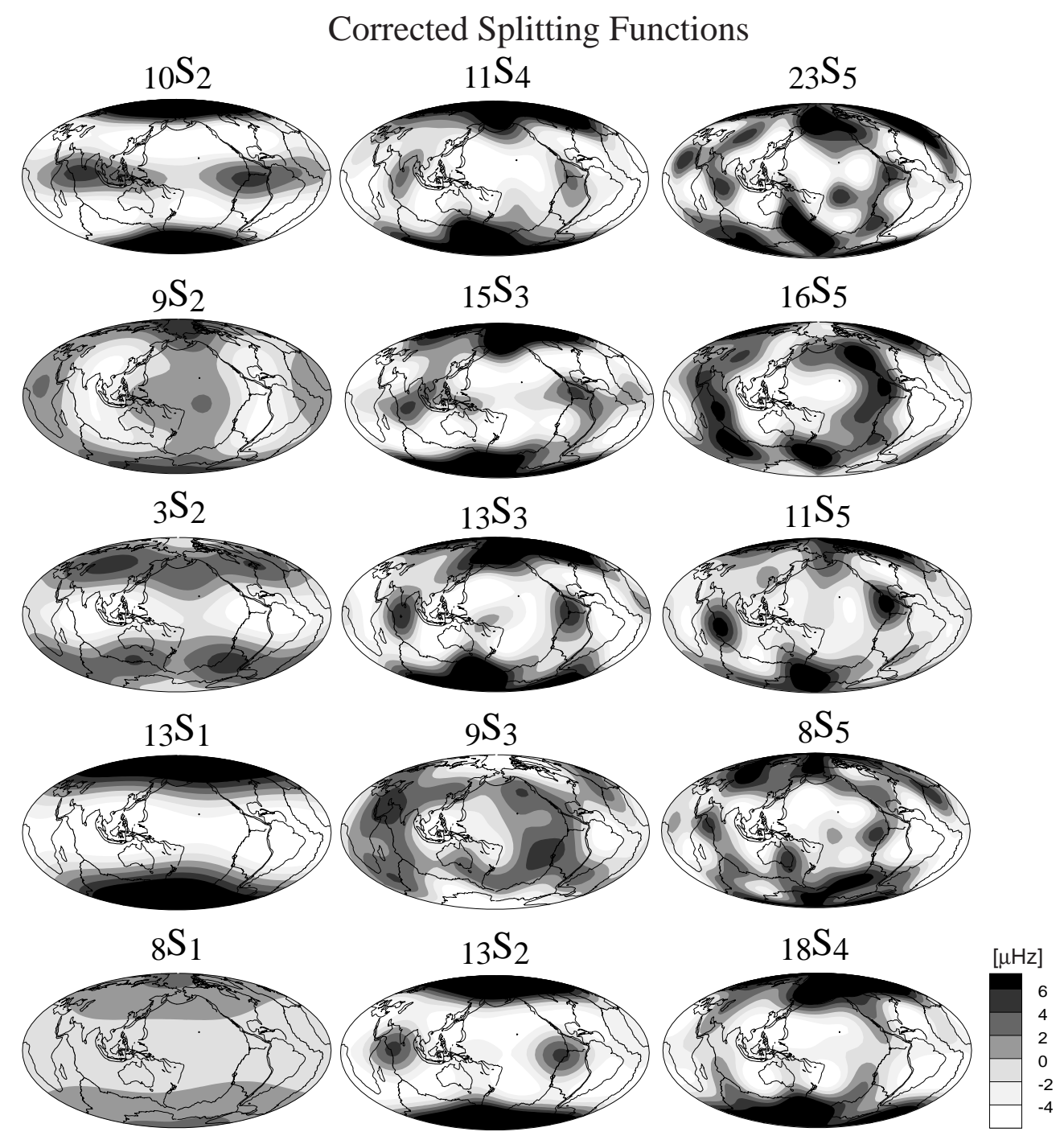

Figure 13. Observed splitting functions, after correction for crustal and mantle signal. 


\section{Misfit as Function of Rotation Angle for 13S2}

Events which Pass the Selection Process
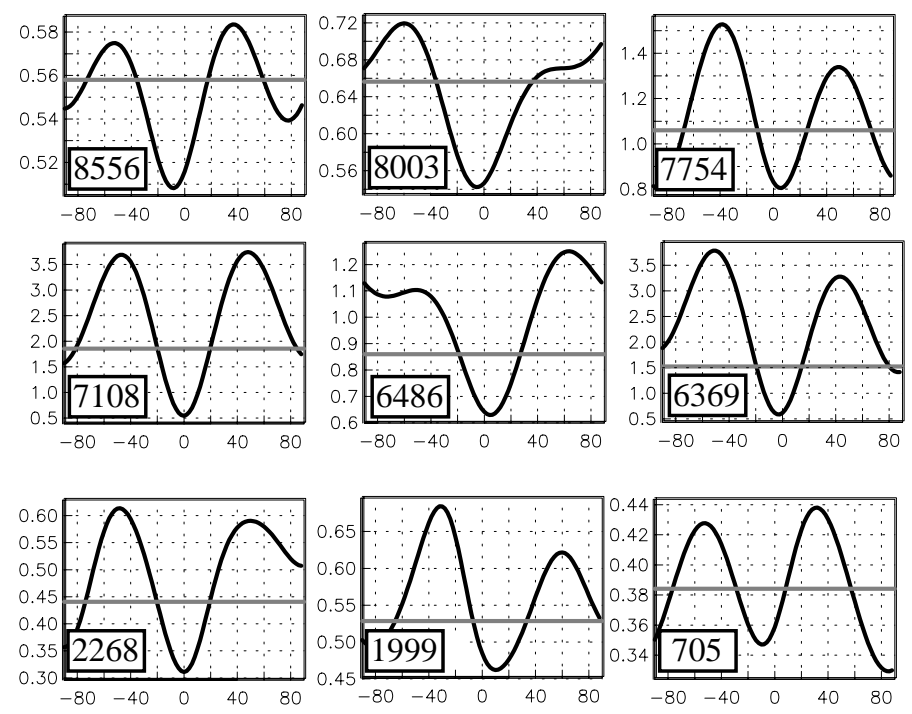

Events which Fail the Selection Process

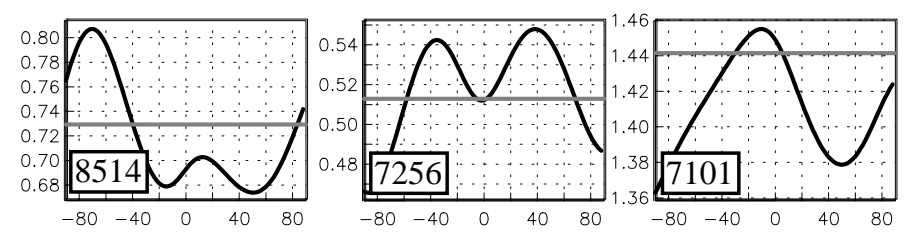

Figure 14. Examples for misfit functions for mode ${ }_{13} S_{2}$ of events which pass the selection process and which fail. The horizontal grey line marks the fit for the zonal component and the number in the lower left corner in each diagram is the day since Jan. 01, 1977, e.g. day 6369 is the Bolivia event (see Table 1). 


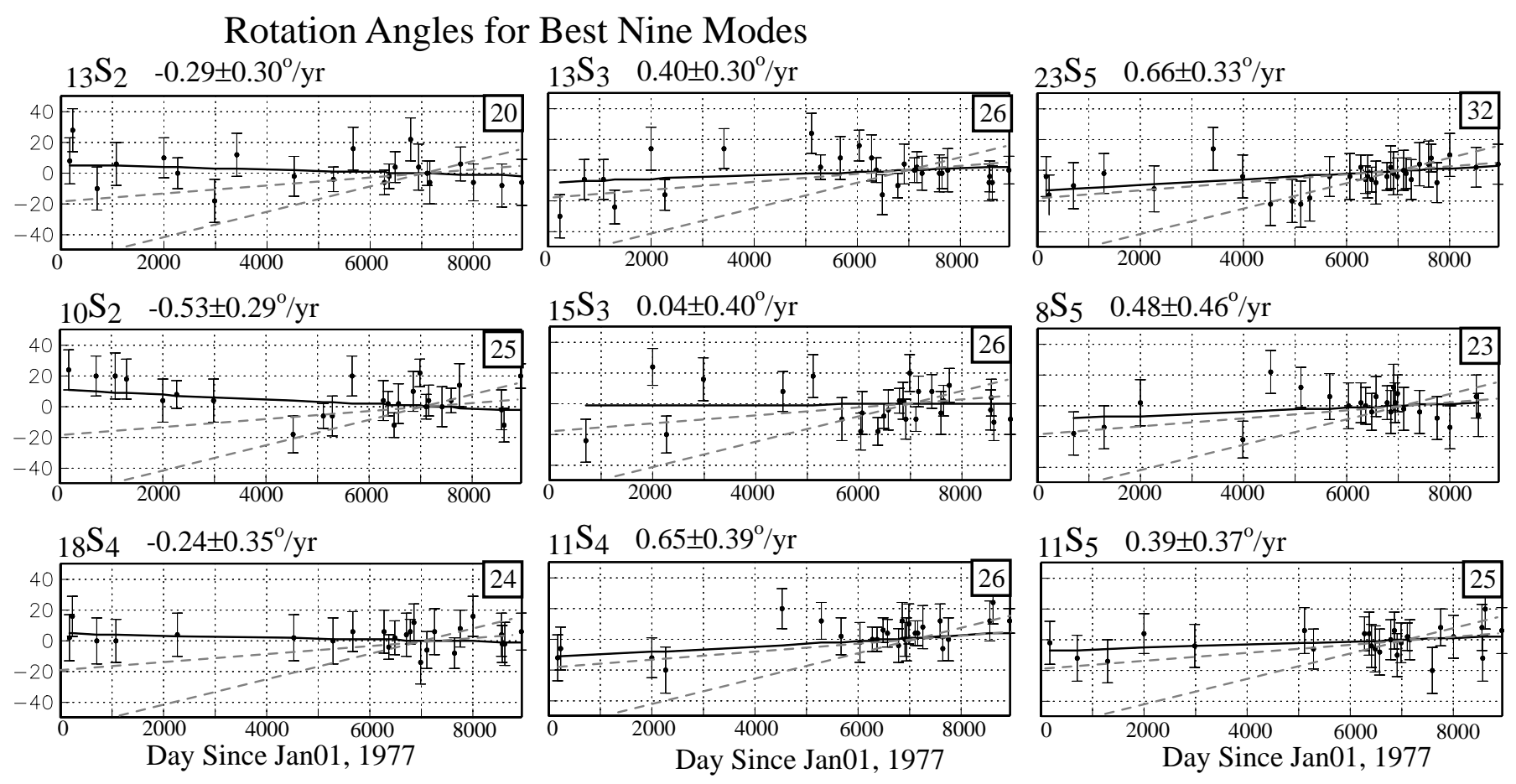

Figure 15. Rotation angles and inferred differential rotation rate for the "best nine modes" (i.e. high number of events). The solid line marks the best fitting straight line that has zero angle at the median time for which the "recent" splitting function was determined. Grey dashed lines mark assumed rotation rates of $1^{\circ}$ and $2^{\circ}$ per year. For most modes, these high rates are inconsistent with the measured angles. The number of events for each mode is given in the upper right corner. 


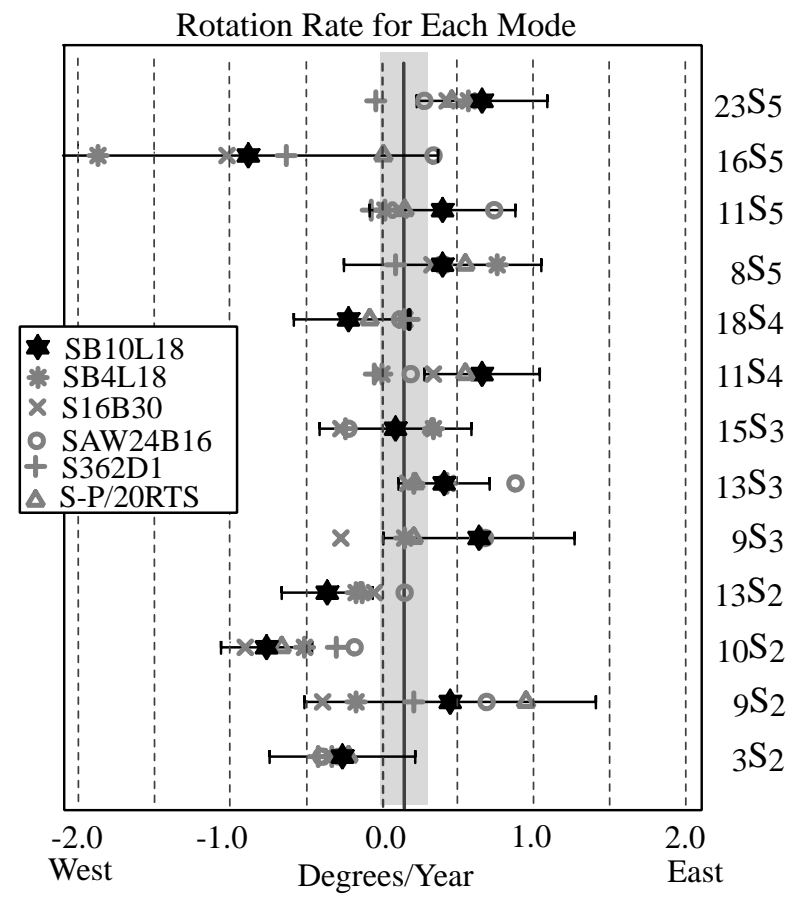

Figure 16. Inner core rotation rates obtained for 13 inner core-sensitive modes, using our preferred mantle model SB10L18. Also shown are the results obtained using other mantle models (for details see Figure 17). The results using different models are remarkably consistent and variations seem to be mode specific, not model specific. The least squares fitting rotation rate, using all modes, is $0.13 \pm 0.11^{\circ} / \mathrm{yr}$ (light grey area). A rotation rate of $0.25^{\circ} / \mathrm{yr}$ is therefore marginally consistent with our data. 


\section{Misfit Function for 13S3 for the Bolivia Event}
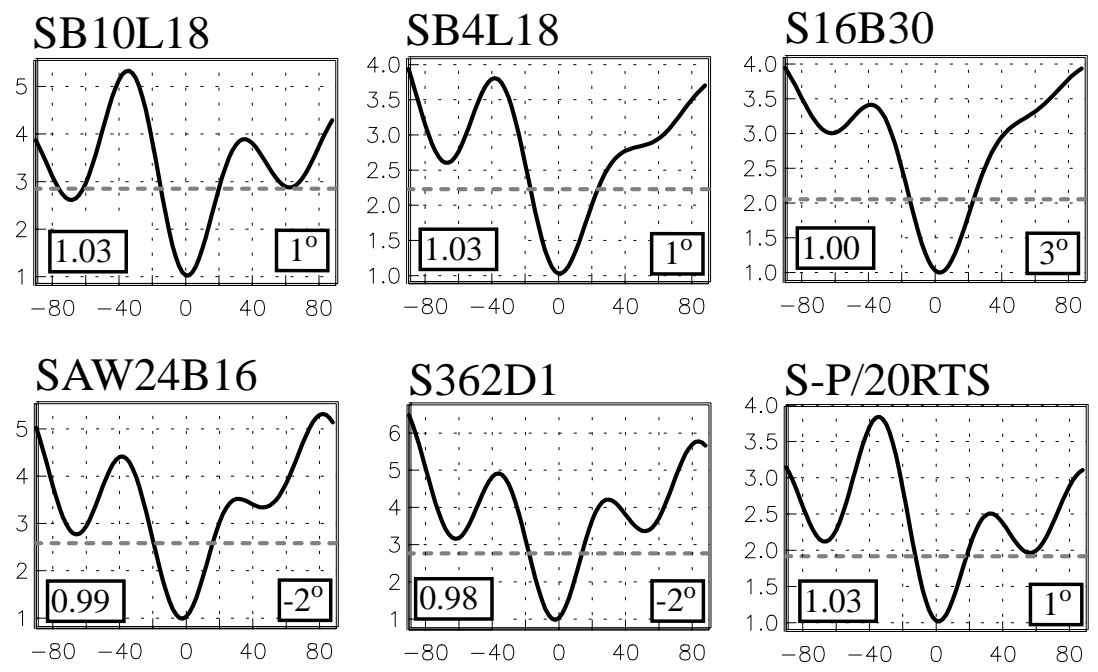

Figure 17. Misfit function for mode ${ }_{13} S_{3}$ for the 94 Bolivia Event obtained for different mantle models. Joint Vs/Vc or Vs/Vp models: SB10L18: Masters et al. (2000b); S-P/20RTS: Ritsema and van Heijst (2000). Shear velocity models: SB4L18: Masters et al. (2000b); S16B30: Masters et al. (1996); SAW24B16: Megnin and Romanowicz (2000); S362D1: Gu and Dziewonski (1999). The minimum misfit as well as the optimal rotation angle are given. 
Table 1. Earthquakes used in this Study

\begin{tabular}{|c|c|c|c|c|c|}
\hline Event Name & Year.Day & $\begin{array}{r}\text { Depth } \\
{[\mathrm{km}]}\end{array}$ & $\begin{array}{l}\text { Moment } \\
{\left[10^{20} \mathrm{Nm}\right]}\end{array}$ & $\begin{array}{l}\text { No. of } \\
\text { records }\end{array}$ & $\begin{array}{r}\text { Day since } \\
1 \text { Jan. } 1977\end{array}$ \\
\hline Arequipa/South. Peru & 2001.174 & 26 & 49 & 85 & 8940 \\
\hline Bonin Islands Region & 2000.219 & 394 & 1.2 & 120 & 8619 \\
\hline South Indian Ocean & 2000.170 & 10 & 7.9 & 133 & 8570 \\
\hline Southern Sumatera & 2000.156 & 33 & 7.5 & 130 & 8556 \\
\hline Santiago del Estero, Argentina & 2000.114 & 608 & 0.3 & 83 & 8514 \\
\hline USSR/China Border & 1999.098 & 566 & 0.5 & 118 & 8133 \\
\hline Molucca Sea/Ceram Sea & 1998.333 & 33 & 4.5 & 71 & 8003 \\
\hline Balleny Islands Region & 1998.084 & 33 & 18.2 & 81 & 7754 \\
\hline Kamchatka & 1997.339 & 33 & 5.3 & 110 & 7644 \\
\hline Fiji Islands & 1997.287 & 166 & 4.6 & 88 & 7592 \\
\hline Santa Cruz Islands & 1997.111 & 33 & 4.4 & 93 & 7416 \\
\hline Peru & 1996.317 & 33 & 4.6 & 97 & 7256 \\
\hline Fiji Islands & 1996.218 & 550 & 1.4 & 96 & 7157 \\
\hline Flores & 1996.169 & 587 & 7.3 & 90 & 7108 \\
\hline Andreanof Isl., Aleutians & 1996.162 & 33 & 8.1 & 97 & 7101 \\
\hline Irian Jaya & 1996.048 & 33 & 24.1 & 103 & 6987 \\
\hline Minahassa Penins., Celebes & 1996.001 & 24 & 7.8 & 91 & 6940 \\
\hline Kuril Islands & 1995.337 & 33 & 8.2 & 83 & 6911 \\
\hline Jalisco, Mexico & 1995.282 & 33 & 11.5 & 96 & 6856 \\
\hline Chile & 1995.211 & 46 & 12.2 & 111 & 6785 \\
\hline Loyalty Islands & 1995.136 & 20 & 3.9 & 80 & 6710 \\
\hline Honshu & 1994.362 & 26 & 4.9 & 87 & 6571 \\
\hline Kuril Islands & 1994.277 & 54 & 30.0 & 101 & 6486 \\
\hline Hokkaido & 1994.202 & 471 & 1.1 & 76 & 6411 \\
\hline Bolivia & 1994.160 & 631 & 26.3 & 88 & 6369 \\
\hline Java & 1994.153 & 18 & 5.3 & 85 & 6362 \\
\hline Fiji Islands & 1994.068 & 562 & 3.1 & 83 & 6277 \\
\hline South of Mariana Isl. & 1993.220 & 59 & 5.2 & 72 & 6064 \\
\hline Hokkaido & 1993.193 & 16 & 4.7 & 72 & 6037 \\
\hline Fiji Islands & 1992.193 & 377 & 0.8 & 65 & 5671 \\
\hline Bolivia & 1991.174 & 558 & 0.9 & 56 & 5287 \\
\hline New Britain & 1990.364 & 178 & 1.8 & 44 & 5112 \\
\hline Philippines & 1990.197 & 25 & 4.1 & 43 & 4945 \\
\hline Sakhalin Island & 1990.132 & 605 & 0.8 & 41 & 4880 \\
\hline Macquarie Islands & 1989.143 & 10 & 13.6 & 52 & 4526 \\
\hline Alaska & 1987.334 & 10 & 7.3 & 41 & 3986 \\
\hline Andreanof Isl.,Aleutians & 1986.127 & 33 & 10.4 & 42 & 3414 \\
\hline Chile & 1985.062 & 33 & 10.3 & 29 & 2984 \\
\hline New Ireland & 1983.077 & 70 & 4.6 & 32 & 2268 \\
\hline Banda Sea & 1982.173 & 450 & 1.8 & 21 & 1999 \\
\hline New Hebrides & 1980.199 & 33 & 4.8 & 21 & 1294 \\
\hline Colombia & 1979.346 & 24 & 16.9 & 26 & 1076 \\
\hline Kuril Islands & 1978.340 & 91 & 6.4 & 24 & 705 \\
\hline Sumbawa & 1977.231 & 33 & 35.9 & 18 & 231 \\
\hline Tonga Islands & 1977.173 & 65 & 13.9 & 16 & 173 \\
\hline
\end{tabular}

